\title{
Are metallic A-F giants evolved Am stars? Rotation and rate of binaries among giant $\mathbf{F}$ stars $^{\star, \star \star}$
}

\author{
M. Künzli and P. North \\ Institut d'Astronomie de l'Université de Lausanne, CH-1290 Chavannes-des-Bois, Switzerland
}

Received October 30, 1996; accepted May 9, 1997

\begin{abstract}
We test the hypothesis of Berthet (1992) which foresees that Am stars become giant metallic A and $\mathrm{F}$ stars (defined by an enhanced value of the blanketing parameter $\Delta m_{2}$ of the Geneva photometry) when they evolve.

If this hypothesis is right, Am and metallic A-FIII stars need to have the same rate of binaries and a similar distribution of $v \sin i$. From our new spectroscopic data and from $v \sin i$ and radial velocities in the literature, we show that it is not the case. The metallic giant stars are often fast rotators with $v \sin i$ larger than $100 \mathrm{~km} \mathrm{~s}^{-1}$, while the maximum rotational velocity for Am stars is about $100 \mathrm{~km} \mathrm{~s}^{-1}$. The rate of tight binaries with periods less than 1000 days is less than $30 \%$ among metallic giants, which is incompatible with the value of $75 \%$ for Am stars (Abt \& Levy 1985). Therefore, the simplest way to explain the existence of giant metallic F stars is to suggest that all normal A and early F stars might go through a short "metallic" phase when they are finishing their life on the main sequence.

Besides, it is shown that only giant stars with spectral type comprised between $\mathrm{F} 0$ and $\mathrm{F} 6$ may have a really enhanced $\Delta m_{2}$ value, while all A-type giants seem to be normal.
\end{abstract}

Key words: stars: binaries: spectroscopic - stars: chemically peculiar — stars: evolution - stars: rotation - stars: $\delta$ Scuti

\section{Introduction}

Main sequence $\mathrm{A}$ and $\mathrm{F}$ stars show various interesting phenomena: chemical peculiarities (Am, Fm, Ap...) on the

Send offprint requests to: P. North

* Based on observations collected at Observatoire de Haute Provence (OHP), France.

** Tables 2, 3, 6 are also available in electronic form at CDS via anonymous ftp to cdsarc.u-strasbg.fr (130.79.128.5) or via http://cdsweb.u-strasbg.fr/Abstract.html one hand and/or pulsation on the other hand. Numerous studies have been devoted to this part of the HR diagram near the ZAMS, but few have explored more evolved stars. In this paper, we examine the rate of binaries and rotation of giant $\mathrm{A}$ and $\mathrm{F}$ stars.

In a study of 132 giant A and F stars, Hauck (1986) showed that $36 \%$ of them have an enhanced value of the blanketing parameter $\Delta m_{2}$ (defined roughly speaking by $m_{2}$ (observed) $-m_{2}$ (Hyades) $)$ characterising Am and $\delta$ Del stars. He showed also that this parameter could probably be interpreted in terms of metallicity for giant $\mathrm{A}$ and $\mathrm{F}$ stars. This interpretation was confirmed by Berthet (1990, 1991) from a detailed abundance analysis of such objects. His study pointed out that these stars have chemical properties similar to those of $\delta$ Del stars, i.e. an overabundance of iron peak elements and especially of heavier elements such as $\mathrm{Sr}$ and $\mathrm{Ba}$, and solar composition for $\mathrm{Ca}$ and Sc. Am stars have similar characteristics for iron peak elements, but $\mathrm{Ca}$ and $\mathrm{Sc}$ are deficient by factors of about 10 .

Based on chemical abundances, position in the $\left(\beta, M_{v}\right)$ plane of Strömgren photometry, rotation and duplicity of $\delta$ Del stars, Kurtz (1976) suggested that these stars are evolved Am stars. The fact that $\delta$ Del and giant metallic A and $\mathrm{F}$ stars harbour similar chemical properties suggests also a link between Am and giant metallic A and F stars. Thus, a star could begin its life on the main sequence as an Am star, then, as abundances of $\mathrm{Ca}$ and Sc increase to quasi-solar values with evolution, it would become a $\delta$ Del star and finally a giant metallic A-F where $\mathrm{Ca}$ and Sc are solar (Berthet 1992).

To explain the emergence of chemical anomalies in Am stars, one generally invokes the radiative diffusion theory developed by Michaud et al. (1983). This theory predicts that, in slow rotators, helium is no longer sustained and flows inside the star and gradually disappears from the atmosphere. The diffusion process could therefore take place just below the thin $\mathrm{H}$ convective zone where the diffusion time is short with respect to the stellar lifetime; the chemical elements whose radiative acceleration is larger than gravity become overabundant and, in the opposite 
case, underabundant. The convective zone becomes deeper with evolution and so leads to normalisation of the surface abundance. This theory explains the chemical anomalies in Am stars and the increase of $\mathrm{Ca}$ and $\mathrm{Sc}$ abundances with time.

The chemical anomalies predicted by the diffusion theory are generally larger than the observed anomalies. These differences come from uncertainties of the atomic data but also from some mechanism in the stellar atmosphere. The mass loss probably takes a prominent part: a rate of about $10^{-15} M_{\odot} /$ year is sufficient to reduce the theoretical overabundance of heavy elements to observed abundances (Charbonneau \& Michaud 1991). Fast rotation $\left(v \sin i>120 \mathrm{~km} \mathrm{~s}^{-1}\right)$ generates meridional circulation which prevents the disappearance of the helium ionisation zone, so the metallic anomalies cannot appear (Michaud 1983). Observationally, the upper limit of rotation for Am stars is about $100 \mathrm{~km} \mathrm{~s}^{-1}$ (Abt \& Moyd 1973), while for normal stars, we observe projected rotational velocities larger than $100 \mathrm{~km} \mathrm{~s}^{-1}$, which is in agreement with the diffusion theory. The meridional circulation cannot be invoked to reduce theoretical abundances to observed ones: in some cases, they can increase the theoretical abundances and qualitatively no relation exists between $v \sin i$ and the chemical anomalies in the velocity range characteristic of Am and Fm stars $\left(0-100 \mathrm{~km} \mathrm{~s}^{-1}\right)$. Indeed, the time diffusion under the hydrogen convective zone is shorter than that of the meridional circulation.

The rate of binaries is completely different in Am and normal stars: Am stars are often members of tight binaries ( $P \leq 100$ days), while normal stars in double systems often have larger periods $(P>100$ days $)$. So, we would be tempted to explain the low rotation of Am stars by tidal braking. Nevertheless, Zahn (1977) showed that this effect is really important only for tight binaries with a period less than 7 days. On the other hand, Abt \& Levy (1985) showed that $75 \%$ of Am stars have periods below 1000 days, so they do not necessarily belong to tight binaries. Other mechanisms must contribute to reduce the rotational velocity to $100 \mathrm{~km} \mathrm{~s}^{-1}$ or less. One of them may be the evolutionary expansion of stars during their main sequence lifetime. According to Abt \& Levy (1985), during this phase, the velocity decreases by a factor of 2 and consequently most of the normal A stars may become Am stars before leaving the main sequence. These authors suggest also the possibility of tidal braking during the premain sequence phase to explain the exclusion of normal stars with a period comprised between 10 and 100 days.

The goal of the present work is to compare the $v \sin i$ and the rate of binaries among Am and giant metallic A and $\mathrm{F}$ stars in order to consider the possibility of a link between these two types of stars. To this end, we have measured at OHP some of Hauck's stars having no radial velocities or $v \sin i$, or only old determinations. These data should allow to strengthen a preliminary work (North
1994) which casts some doubts on the validity of the scenario advocated by Berthet (1992).

\section{The sample and the observations}

We defined the sample to be observed from Table 3 and Table 4 of Hauck (1986). The reason why we considered Table 4 (containing stars classified spectroscopically as dwarfs but photometrically as giants) was that the photometric criterion of luminosity seems much more reliable than the spectroscopic luminosity class.

The selection criteria were:

\section{- Visibility from OHP $\left(\delta \gtrsim-20^{\circ}\right)$ \\ - Insufficient $V_{\mathrm{r}}$ data or unknown $v \sin i$.}

By insufficient $V_{\mathrm{r}}$, we mean that the star has less than three published $V_{\mathrm{r}}$ values in the literature. Applying these criteria, $50 \mathrm{~A}$ and $\mathrm{F}$ giant stars were selected, both normal and metallic. It was judged useful to have a good estimate of the binary freqency of normal $\mathrm{A}$ and $\mathrm{F}$ giants for reference purposes. 40 stars in the sample are non-metallic, while only ten are metallic according to the criterion $\Delta m_{2} \geq 0.013$ (see Sect. 5.1). Thus, the estimate of binary frequency among metallic giants will mainly rely upon old, published $V_{\mathrm{r}}$ data. The $\Delta m_{2}$ values of the sample stars range between -0.027 and 0.082 . They have been updated using a new reference sequence in the $m_{2} / B 2-V 1$ diagram (Hauck et al. 1991), and sometimes by complementary photometric measurements. Eight stars belong to the $\delta$ Scuti class, and nine are spectroscopically classified as dwarf but have a large $\Delta d$ parameter $(\Delta d$ is the luminosity parameter of Geneva photometry, equivalent to Strömgren's $\delta c_{1}$ ) indicating that they very probably are real giants. All stars are bright, with $V \leq 7.0$, implying that interstellar reddening is insignificant.

The observations were performed at the Observatoire de Haute Provence (OHP) with the Aurélie spectrograph attached to the $1.52 \mathrm{~m}$ telescope at the Coudé focus (Gillet et al. 1994) in 1994. Four runs of a few nights' duration each were made respectively at the beginning of May and June for 18 stars and at the beginning of November and December for 32 stars. These measurements should allow to detect binary stars with a small period ( $P \lesssim 100$ days).

The detector is a double barrette CCD Thomson TH7832 with 2048 pixels having a size of $750 \times 13 \mu \mathrm{m}$. The spectra were obtained at a reciprocal dispersion of $8 \AA \mathrm{mm}^{-1}$ in the spectral region centred on $\mathrm{H} \beta$ [4780 ̊, $5000 \AA]$. The reduction was made at the OHP with IHAP procedures, using comparison spectra of thorium. Each stellar measurement was preceded by a calibration exposure to compensate for instrumental drift as much as possible. To normalise our spectrum, we simply fit a straight line to the continuum. 291 stellar exposures were made, of which 54 were devoted to standard stars. For most of the spectra a signal-to-noise of 150 was achieved. The minimum number of exposures per star was 2 and the 
maximum 11 , with a mean value of 5 or 6 measurements per star.

\section{Radial velocity determinations}

\subsection{H $\beta$ fitted by a lorentz profile}

Some stars of the sample rotate very fast and some are very hot, with effective temperature of about $8000 \mathrm{~K}$. Therefore, these stars cannot be measured with an instrument such as Coravel, which needs many narrow lines. The adopted technique consists in fitting a lorentz profile on the $\mathrm{H} \beta$ line $[4861.331 \AA]$ minimising the $\chi^{2}$, i.e. the differences between the lorentz profile and the observed line. The advantage of a lorentz function over a gaussian is to be more peaked at the centre, so hydrogen lines are better fitted.

A lorentz profile is defined by 3 parameters (Eq. 1): $\lambda_{0}$ is the centre, $i_{0}$ the intensity or height and $b$ the halfintensity width of the profile.

$L(\lambda)=\frac{i_{0} b^{2}}{\left(\lambda-\lambda_{0}\right)^{2}+b^{2}}$.

The three parameters of $L(\lambda)$ are fitted by least-squares; meanwhile the optimum choice will depend on the limits fixing the spectral range on which the fit is made. To avoid too subjective a choice, we fix on both sides of the line two points determining a segment on which the limit is randomly chosen (Fig. 1). Thus, we generate 100 lorentz profiles with different limits on each $\mathrm{H} \beta$ line. A profile is taken into account only if the $\chi^{2}$ value does not exceed 0.05 in order to avoid hazardous fits. Then, we define for each line $\mathrm{H} \beta$ the mean value of the central wavelength and the dispersion:

$\overline{\lambda_{0}(\mathrm{H} \beta)}=\frac{1}{n} \sum_{i=i}^{n} \lambda_{0}(\mathrm{H} \beta)_{i}, n=100$

$\sigma_{\lambda_{0}(\mathrm{H} \beta)}=\sqrt{\sum_{i=i}^{n} \frac{1}{n}\left(\lambda_{0}(\mathrm{H} \beta)_{i}-\overline{\lambda_{0}(\mathrm{H} \beta)}\right)^{2}}, n=100$.

The dispersion $\sigma_{\lambda_{0}(\mathrm{H} \beta)}$ depends on the physical properties of the star, essentially the temperature, the surface gravity and the rotation but also the quality of the spectrum, i.e. the signal-to-noise. We have chosen to distribute the $V_{\mathrm{r}}$ measurements into categories of decreasing discrete precision on the basis of $\sigma_{\lambda_{0}(\mathrm{H} \beta)}$ values (see Table 1): measurements with $\sigma_{\lambda_{0}(\mathrm{H} \beta)}$ between 0 and $3 \mathrm{m \AA}$ will be considered as having $\sigma_{V_{\mathrm{r}}}^{\mathrm{s}}=0.19 \mathrm{~km} \mathrm{~s}^{-1}$, those with $\sigma_{\lambda_{0}(\mathrm{H} \beta)}$ between 3 and $6 \mathrm{m \AA}$ will have $\sigma_{V_{\mathrm{r}}}^{\mathrm{s}}=0.38 \mathrm{~km} \mathrm{~s}^{-1}$, etc. The value of $3 \mathrm{~m} \AA$ is completely arbitrary, corresponding to a radial velocity of about $0.2 \mathrm{kms}^{-1}$. The $\sigma_{\lambda_{0}(\mathrm{H} \beta)}$ and the central wavelength $\overline{\lambda_{0}(\mathrm{H} \beta)}$ also depend on the choice of the limits for the fit. This effect is discussed in detail in Appendix A.
Table 1. Dispersion in radial velocity $\sigma_{V_{\mathrm{r}}}^{\mathrm{s}}$ from the dispersion in wavelength $\sigma_{\lambda_{0}(\mathrm{H} \beta)}$

\begin{tabular}{lc}
\hline$\sigma_{\lambda_{0}(\mathrm{H} \beta)}[\AA]$ & $\sigma_{V_{\mathrm{r}}}^{\mathrm{s}}\left[\mathrm{km} \mathrm{s}^{-1}\right]$ \\
\hline$[0.000,0.003]$ & 0.19 \\
{$[0.003,0.006]$} & 0.38 \\
{$[0.006,0.009]$} & 0.56 \\
{$[0.009,0.012]$} & 0.74 \\
$\ldots$ & \\
\hline
\end{tabular}

The term $\sigma_{V_{\mathrm{r}}}^{\mathrm{s}}$ is defined as the internal error due to the effective temperature, the surface gravity, the rotation and the $S / N$ ratio. The total internal error includes an additional term due to the instrumental drift during the night. This variability was similar for the four missions and Figs. 2 and 3 show the fluctuations of the position of the thorium lines during the night of 6 and 7 November and during the night of 5 and 6 December 1994 for 3 lines. When we filled up the nitrogen tank, at the beginning and in the middle of the night, a strong variation appeared as shown on these figures. Between these jumps, we observe a regular variation of about 0.12 pixels per hour. We therefore have a variation of $0.16 \mathrm{~km} \mathrm{~s}^{-1}$ per 12 minutes which corresponds to the average time of stellar exposure. For this reason, the internal error due to this factor $\left(\sigma_{V_{\mathrm{r}}}^{\mathrm{t}}\right)$ is estimated at $0.16 \mathrm{~km} \mathrm{~s}^{-1}$. Finally, the total internal error is written by $I=\sqrt{\left(\sigma_{V_{\mathrm{r}}}^{\mathrm{s}}\right)^{2}+\left(\sigma_{V_{\mathrm{r}}}^{\mathrm{t}}\right)^{2}}$.

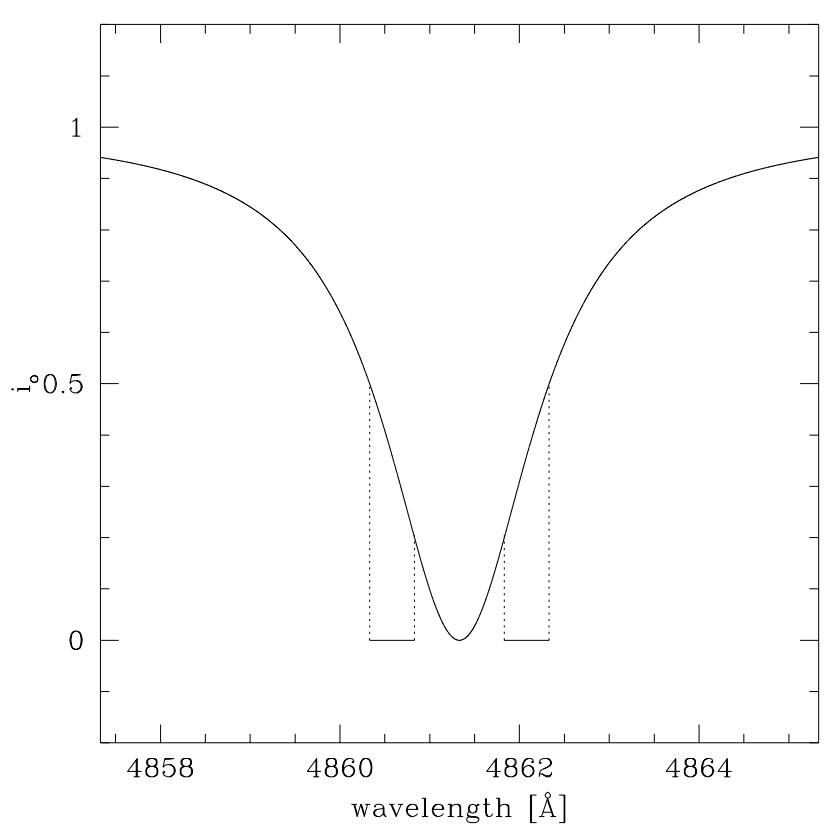

Fig. 1. The limits fixing the spectral range on which the fit is made are randomly chosen on two segments symmetrically placed with respect to the centre of the line 


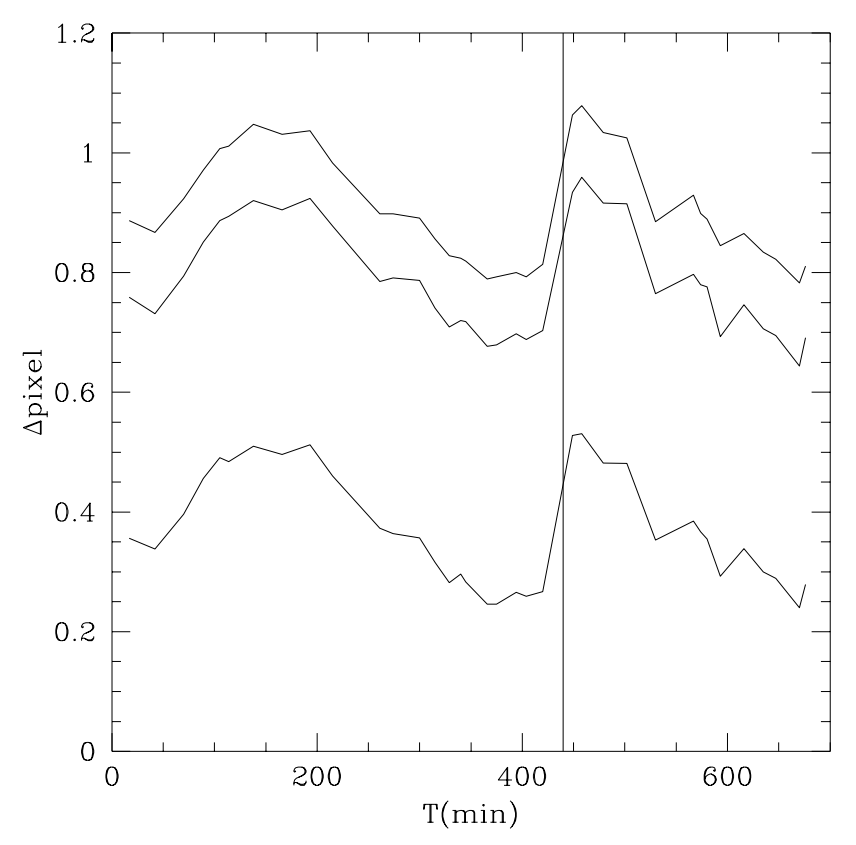

Fig. 2. Variation of the position of three lines of thorium during 6 and 7 November 1994. The vertical shift is arbitrary. The vertical line corresponds to the filling of nitrogen

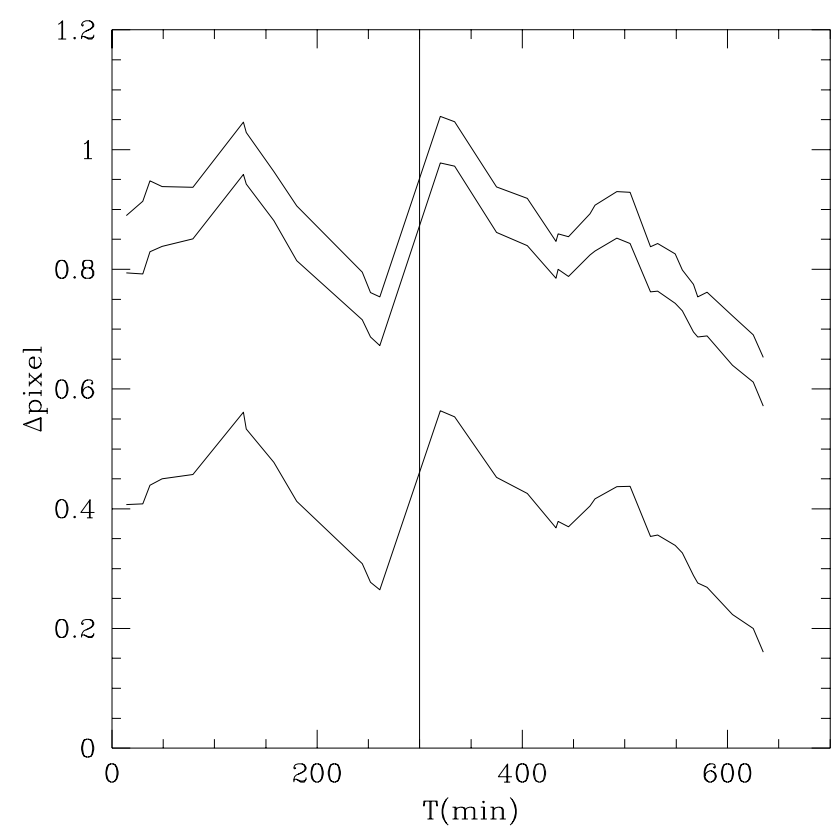

Fig. 3. Same as Fig. 2, but for the night of 5 and 6 December 1994

\subsection{Standard stars}

For standard stars, we prefer to use Coravel values in the system of faint IAU standard stars (Mayor \& Maurice 1985) than IAU values listed in the Astronomical Almanach because the latter are taken from various authors and sometimes not updated. For example, the IAU radial velocity for HD 114762 has been listed as $49.9 \pm$ $0.5 \mathrm{~km} \mathrm{~s}^{-1}$ in the Astronomical Almanach since at least 1981, while combined data from the Cfa and Coravel give a systemic velocity of $49.35 \pm 0.04 \mathrm{~km} \mathrm{~s}^{-1}$ (Latham et al. 1989).

For the four observing runs, Fig. 4 shows the difference between radial velocities measured with Aurélie and the Coravel value for each standard star. Our values are higher than the Coravel ones by about $2.75 \mathrm{~km} \mathrm{~s}^{-1}$. The error bars are defined by the quadratic sum of the internal error (see Sect. 3.1) and the dispersion on the Coravel values. The internal precision of our measurements is very impressive: we obtain a scatter of about $0.4 \mathrm{~km} \mathrm{~s}^{-1}$ around the mean radial velocity.
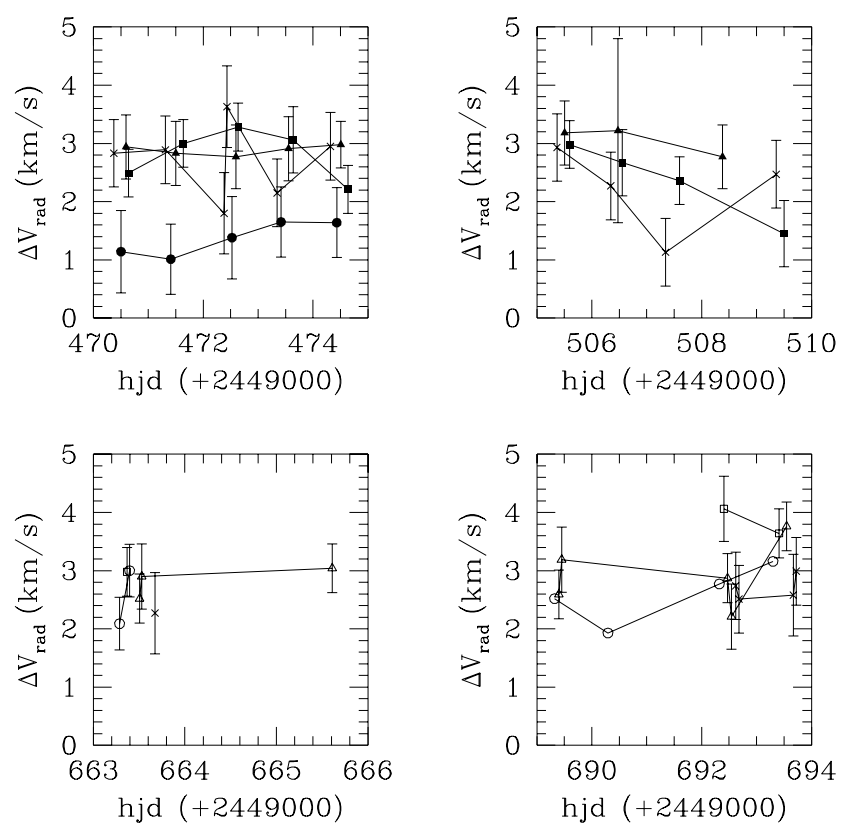

Fig. 4. Difference between Aurélie radial velocities and Coravel velocities for the runs of May, June, November and December 1994. HD 693: open squares. HD 22484: open triangles. HD 89449: crosses. HD 114762: full circles. HD 136202: full triangles. HD 222368: open circles

Notice that $\Delta V_{\mathrm{r}}$ is clearly smaller for HD 114762 by about $1.4 \mathrm{~km} \mathrm{~s}^{-1}$. Such a difference can partly be due to the nature of this star, which has a very small amplitude (Latham et al. 1989 and Cochran et al. 1991) and to the fact that our measurements were made precisely when the radial velocity was minimum: in this way we can explain 
a shift of about $0.6 \mathrm{~km} \mathrm{~s}^{-1}$ with respect to the other standard stars. Unfortunately, we do not find any explanation for the remaining shift of $0.8 \mathrm{~km} \mathrm{~s}^{-1}$. The reduction of the spectra was made again with MIDAS software, only to find the same shift. So, we cannot question the reduction.

We have simply subtracted the mean $\Delta V_{\mathrm{r}}$ from the measured velocities. The average $\Delta V_{\mathrm{r}}$ are 2.80, 2.49, 2.69 and $2.90 \mathrm{~km} \mathrm{~s}^{-1}$ for the runs of May, June, November and December respectively. For the first run, the values of HD 114762 are not taken into account in the average value. Table 2 gives the individual corrected radial velocities and the mean velocity for standard stars; Table 3 lists the individual corrected radial velocities for the 50 giant $\mathrm{A}$ and F stars.

\section{Binarity among the sample}

\subsection{Criterion of variability}

To take into account the errors of measurements on the determination of the duplicity, we computed the $\chi^{2}$ value for each star of the programme: $\chi^{2}=(n-1)\left(\frac{E}{I}\right)^{2}$ where $n$ is the number of measurements, $E$ the external error and $I$ the internal error. We then used an F-test which gives the probability $P\left(\chi^{2}\right)$ that the variations of velocity are only due to the internal dispersion. A star will be considered as double or intrinsically variable if $P\left(\chi^{2}\right)$ is less than 0.01 (Duquennoy \& Mayor 1991). Naturally, this test cannot say anything about the nature of the variability.

The distribution of $P\left(\chi^{2}\right)$ for non-variable stars should be flat from 0 to 1 , while the variable stars should gather at the smallest values of $P\left(\chi^{2}\right)$. Therefore, this method allows to appreciate a posteriori the estimate of internal errors. Indeed, if these errors are underestimated, a gradient appears in the distribution of $P\left(\chi^{2}\right)$ in favour of small values, while if they are overestimated, a peak appears near 1 , indicating an abnormally strong predominance of constant stars.

In our case, the internal error is estimated by the quadratic sum of a term which depends on the width of the $\mathrm{H} \beta$ line and the quality of the spectrum, and a second term related with the instrumental shift during the night. While the last term is rather well controlled, the first one is not well-known. Indeed, it strongly depends on the choice of the limits (see Sect. 3.2). The internal error can be written:

$I=\sqrt{\left(\alpha \sigma_{V_{\mathrm{r}}}^{\mathrm{s}}\right)^{2}+\left(\sigma_{V_{\mathrm{r}}}^{\mathrm{t}}\right)^{2}}$

where $\sigma_{V_{\mathrm{r}}}^{\mathrm{s}}$ is the dispersion due to $T_{\text {eff }}, v \sin i$ and $S / N$, $\sigma_{V_{\mathrm{r}}}^{\mathrm{t}}$ is the dispersion due to instrumental drift and $\alpha$ is adjusted to obtain a flat distribution of $P\left(\chi^{2}\right)$ on the interval $[0,1]$, except for the small values of course. $\alpha$ indicates the quality of the preliminary estimation of $I$. To determine $\alpha$ quantitatively, one uses the cumulative distribution of $P\left(\chi^{2}\right)$ which must approximate a straight line in the case of a flat distribution. For a given $\alpha$, one can
Table 2. Radial velocities of standard stars

\begin{tabular}{|c|c|c|c|}
\hline $\mathrm{HD}$ & $\begin{array}{c}\text { HJD } \\
(+2449000)\end{array}$ & $\begin{array}{r}V_{\mathrm{r}}^{\mathrm{OHP}} \\
\mathrm{km} \mathrm{s}^{-1}\end{array}$ & $\begin{array}{c}I \\
\mathrm{~km} \mathrm{~s}^{-1}\end{array}$ \\
\hline 693 & 663.367 & 15.12 & 0.28 \\
\hline 693 & 692.408 & 15.99 & 0.48 \\
\hline \multirow[t]{2}{*}{693} & 693.411 & 15.57 & 0.28 \\
\hline & $\overline{V_{\mathrm{r}}}=$ & $15.44 \pm 0.36$ & \\
\hline 22484 & 663.507 & 27.59 & 0.28 \\
\hline 22484 & 663.528 & 27.97 & 0.48 \\
\hline 22484 & 665.610 & 28.11 & 0.28 \\
\hline 22484 & 689.403 & 27.45 & 0.28 \\
\hline 22484 & 689.450 & 28.05 & 0.48 \\
\hline 22484 & 692.473 & 27.73 & 0.28 \\
\hline 22484 & 692.544 & 27.07 & 0.48 \\
\hline \multirow[t]{2}{*}{22484} & 693.546 & 28.62 & 0.28 \\
\hline & $\overline{V_{\mathrm{r}}}=$ & $27.86 \pm 0.44$ & \\
\hline 89449 & 470.375 & 6.07 & 0.28 \\
\hline 89449 & 471.311 & 6.13 & 0.28 \\
\hline 89449 & 472.383 & 5.04 & 0.48 \\
\hline 89449 & 472.433 & 6.87 & 0.69 \\
\hline 89449 & 473.347 & 5.39 & 0.28 \\
\hline 89449 & 474.319 & 6.19 & 0.28 \\
\hline 89449 & 505.366 & 6.49 & 0.28 \\
\hline 89449 & 506.346 & 5.83 & 0.28 \\
\hline 89449 & 507.345 & 4.69 & 0.28 \\
\hline 89449 & 509.356 & 6.03 & 0.28 \\
\hline 89449 & 663.674 & 5.62 & 0.48 \\
\hline 89449 & 692.618 & 5.88 & 0.28 \\
\hline 89449 & 692.681 & 5.65 & 0.28 \\
\hline 89449 & 693.668 & 5.72 & 0.48 \\
\hline \multirow[t]{2}{*}{89449} & 693.722 & 6.13 & 0.28 \\
\hline & $\overline{V_{\mathrm{r}}}=$ & $5.84 \pm 0.52$ & \\
\hline 114762 & 470.503 & 47.51 & 0.48 \\
\hline 114762 & 471.411 & 47.38 & 0.28 \\
\hline 114762 & 472.526 & 47.75 & 0.48 \\
\hline 114762 & 473.420 & 48.02 & 0.28 \\
\hline \multirow[t]{2}{*}{114762} & 474.432 & 48.01 & 0.28 \\
\hline & $\overline{V_{\mathrm{r}}}=$ & $47.77 \pm 0.26$ & \\
\hline 136202 & 470.593 & 54.33 & 0.48 \\
\hline 136202 & 471.499 & 54.22 & 0.48 \\
\hline 136202 & 472.597 & 54.16 & 0.48 \\
\hline 136202 & 473.555 & 54.30 & 0.48 \\
\hline 136202 & 474.504 & 54.37 & 0.28 \\
\hline 136202 & 505.503 & 54.89 & 0.48 \\
\hline 136202 & 506.474 & 54.93 & 1.56 \\
\hline \multirow[t]{2}{*}{136202} & 508.381 & 54.48 & 0.48 \\
\hline & $\overline{V_{\mathrm{r}}}=$ & $54.39 \pm 0.27$ & \\
\hline 187691 & 470.642 & -0.58 & 0.28 \\
\hline 187691 & 471.638 & -0.07 & 0.28 \\
\hline 187691 & 472.638 & 0.21 & 0.28 \\
\hline 187691 & 473.640 & -0.01 & 0.48 \\
\hline 187691 & 474.639 & -0.86 & 0.28 \\
\hline 187691 & 505.598 & 0.23 & 0.28 \\
\hline 187691 & 506.558 & -0.08 & 0.48 \\
\hline 187691 & 507.599 & -0.39 & 0.28 \\
\hline \multirow[t]{2}{*}{187691} & 509.501 & -1.30 & 0.48 \\
\hline & $\overline{V_{\mathrm{r}}}=$ & $-0.27 \pm 0.49$ & \\
\hline 222368 & 663.287 & 4.76 & 0.28 \\
\hline 222368 & 663.395 & 5.67 & 0.28 \\
\hline 222368 & 689.321 & 4.98 & 0.48 \\
\hline 222368 & 690.296 & 4.39 & 0.28 \\
\hline 222368 & 692.320 & 5.23 & 0.28 \\
\hline \multirow[t]{2}{*}{222368} & 693.300 & 5.62 & 0.28 \\
\hline & $\overline{V_{\mathrm{r}}}=$ & $5.12 \pm 0.45$ & \\
\hline
\end{tabular}


Table 3. Radial velocities of $\mathrm{A}$ and $\mathrm{F}$ giants

\begin{tabular}{|c|c|c|c|}
\hline HD & $\begin{array}{c}\text { HJD } \\
(+2449000)\end{array}$ & $\begin{array}{r}V_{\mathrm{r}} \\
\mathrm{km} \mathrm{s}^{-1}\end{array}$ & $\begin{array}{c}I \\
\mathrm{~km} \mathrm{~s}^{-1}\end{array}$ \\
\hline 1671 & 663.445 & 10.53 & 0.48 \\
\hline 1671 & 689.354 & 10.55 & 0.28 \\
\hline 1671 & 693.444 & 10.34 & 0.48 \\
\hline 2628 & 663.451 & -10.86 & 0.28 \\
\hline 2628 & 689.366 & -10.67 & 1.12 \\
\hline 2628 & 693.448 & -10.40 & 0.28 \\
\hline 4338 & 663.410 & 2.08 & 0.69 \\
\hline 4338 & 689.381 & -0.47 & 1.34 \\
\hline 4338 & 693.470 & 5.84 & 0.94 \\
\hline 6706 & 663.426 & 23.81 & 0.28 \\
\hline 6706 & 692.487 & 23.57 & 0.69 \\
\hline 10845 & 663.458 & -13.97 & 0.48 \\
\hline 10845 & 689.412 & -13.67 & 0.90 \\
\hline 11522 & 663.436 & 1.16 & 0.69 \\
\hline 11522 & 693.514 & 1.99 & 1.34 \\
\hline 12573 & 663.471 & 8.62 & 0.69 \\
\hline 12573 & 692.522 & 7.38 & 0.90 \\
\hline 17584 & 689.423 & 18.41 & 0.94 \\
\hline 17584 & 663.481 & 18.60 & 0.90 \\
\hline 17584 & 692.387 & 19.42 & 0.69 \\
\hline 17918 & 663.491 & 17.21 & 0.48 \\
\hline 17918 & 663.552 & 18.40 & 0.69 \\
\hline 17918 & 689.434 & 17.11 & 0.90 \\
\hline 17918 & 692.396 & 18.08 & 0.90 \\
\hline 21770 & 663.500 & -46.18 & 0.28 \\
\hline 21770 & 689.444 & -46.35 & 0.48 \\
\hline 21770 & 692.469 & -46.01 & 0.28 \\
\hline 24832 & 663.538 & 20.61 & 1.56 \\
\hline 24832 & 692.433 & 18.20 & 0.90 \\
\hline 24832 & 693.569 & 22.67 & 0.69 \\
\hline 30020 & 663.570 & 34.31 & 0.69 \\
\hline 30020 & 692.567 & 37.19 & 0.48 \\
\hline 30020 & 693.609 & 37.87 & 0.90 \\
\hline 34045 & 663.591 & 29.89 & 0.48 \\
\hline 34045 & 689.467 & 26.19 & 0.94 \\
\hline 34045 & 692.587 & 29.18 & 0.48 \\
\hline 48737 & 663.606 & 26.12 & 0.90 \\
\hline 48737 & 665.620 & 26.30 & 0.48 \\
\hline 48737 & 689.459 & 25.77 & 0.48 \\
\hline 48737 & 692.598 & 27.35 & 0.69 \\
\hline 48737 & 693.630 & 27.56 & 0.48 \\
\hline 50019 & 663.611 & 31.17 & 0.69 \\
\hline 50019 & 664.643 & 30.75 & 0.69 \\
\hline 50019 & 665.626 & 30.30 & 0.69 \\
\hline 50019 & 692.601 & 30.04 & 0.69 \\
\hline 50019 & 693.633 & 32.39 & 0.90 \\
\hline 60489 & 663.617 & 52.67 & 0.69 \\
\hline 60489 & 665.645 & 53.42 & 0.69 \\
\hline 60489 & 692.608 & 30.81 & 0.48 \\
\hline 60489 & 693.650 & 31.65 & 0.28 \\
\hline 62437 & 663.627 & 11.13 & 0.48 \\
\hline 62437 & 665.677 & 14.46 & 1.34 \\
\hline
\end{tabular}

Table 3. continued

\begin{tabular}{|c|c|c|c|}
\hline HD & $\begin{array}{c}\text { HJD } \\
(+2449000)\end{array}$ & $\begin{array}{r}V_{\mathrm{r}} \\
\mathrm{km} \mathrm{s}^{-1}\end{array}$ & $\begin{array}{c}I \\
\mathrm{~km} \mathrm{~s}^{-1}\end{array}$ \\
\hline 62437 & 692.628 & 15.99 & 0.28 \\
\hline 62437 & 693.639 & 14.12 & 0.69 \\
\hline 69997 & 663.642 & 31.67 & 0.28 \\
\hline 69997 & 692.641 & 32.79 & 0.28 \\
\hline 69997 & 692.703 & 33.18 & 0.28 \\
\hline 69997 & 693.661 & 32.59 & 0.28 \\
\hline 82043 & 663.652 & 12.25 & 0.48 \\
\hline 82043 & 692.649 & 1.73 & 0.69 \\
\hline 82043 & 692.712 & 1.41 & 0.69 \\
\hline 82043 & 693.679 & 0.14 & 0.48 \\
\hline 84607 & 663.660 & 13.65 & 0.48 \\
\hline 84607 & 692.660 & 12.25 & 0.69 \\
\hline 84607 & 692.723 & 13.50 & 0.69 \\
\hline 84607 & 693.690 & 13.09 & 0.48 \\
\hline 86611 & 663.688 & 26.47 & 0.90 \\
\hline 86611 & 692.669 & 24.42 & 0.90 \\
\hline 86611 & 692.732 & 25.79 & 0.69 \\
\hline 86611 & 693.699 & 25.04 & 1.34 \\
\hline 89025 & 692.676 & -21.20 & 0.69 \\
\hline 89025 & 693.710 & -22.07 & 0.28 \\
\hline 92787 & 692.689 & 4.47 & 0.90 \\
\hline 92787 & 692.692 & 4.47 & 0.90 \\
\hline 92787 & 693.715 & 3.82 & 0.48 \\
\hline 100418 & 470.406 & 1.02 & 0.69 \\
\hline 100418 & 471.327 & 0.76 & 0.58 \\
\hline 100418 & 471.390 & -0.57 & 0.69 \\
\hline 100418 & 472.398 & 2.28 & 0.28 \\
\hline 100418 & 473.336 & 0.22 & 0.69 \\
\hline 100418 & 474.359 & -1.81 & 0.48 \\
\hline 100418 & 506.372 & -0.28 & 0.28 \\
\hline 103313 & 470.461 & 16.10 & 0.69 \\
\hline 103313 & 471.340 & 16.75 & 0.48 \\
\hline 103313 & 471.463 & 14.71 & 0.28 \\
\hline 103313 & 472.413 & 19.14 & 0.90 \\
\hline 103313 & 473.359 & 17.59 & 0.69 \\
\hline 103313 & 473.440 & 17.88 & 0.90 \\
\hline 103313 & 474.378 & 16.54 & 0.69 \\
\hline 103313 & 474.414 & 16.72 & 0.48 \\
\hline 103313 & 505.387 & 22.17 & 1.56 \\
\hline 103313 & 507.364 & 18.64 & 0.48 \\
\hline 103313 & 507.397 & 15.74 & 2.01 \\
\hline 104827 & 470.475 & 10.21 & 0.48 \\
\hline 104827 & 471.352 & 8.07 & 0.90 \\
\hline 104827 & 471.399 & 7.33 & 0.48 \\
\hline 104827 & 472.443 & 7.70 & 0.48 \\
\hline 104827 & 473.373 & 7.60 & 0.48 \\
\hline 104827 & 473.482 & 8.42 & 0.48 \\
\hline 104827 & 474.332 & 7.37 & 0.69 \\
\hline 104827 & 474.479 & 6.93 & 0.48 \\
\hline 104827 & 506.394 & 8.89 & 0.48 \\
\hline 108382 & 470.486 & -2.33 & 0.48 \\
\hline 108382 & 471.364 & -1.97 & 0.48 \\
\hline 108382 & 471.471 & -1.82 & 0.48 \\
\hline 108382 & 472.457 & -2.86 & 0.69 \\
\hline 108382 & 473.383 & -1.97 & 0.90 \\
\hline 108382 & 473.430 & -2.71 & 0.69 \\
\hline 108382 & 474.345 & -1.55 & 0.90 \\
\hline
\end{tabular}


Table 3. continued

\begin{tabular}{|c|c|c|c|}
\hline HD & $\begin{array}{c}\text { HJD } \\
(+2449000)\end{array}$ & 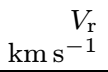 & $\begin{array}{c}I \\
\mathrm{~km} \mathrm{~s}^{-1}\end{array}$ \\
\hline$\overline{118295}$ & 470.535 & -20.00 & 0.69 \\
\hline 118295 & 471.375 & -19.63 & 0.69 \\
\hline 118295 & 471.447 & -17.52 & 1.56 \\
\hline 118295 & 472.470 & -20.59 & 0.69 \\
\hline 118295 & 473.401 & -18.46 & 0.94 \\
\hline 118295 & 474.394 & -17.76 & 0.69 \\
\hline 118295 & 505.410 & -24.23 & 0.94 \\
\hline 118295 & 506.420 & -19.84 & 1.34 \\
\hline 108382 & 474.446 & -1.89 & 0.90 \\
\hline 122703 & 470.522 & -17.63 & 0.69 \\
\hline 122703 & 471.430 & -13.34 & 0.90 \\
\hline 122703 & 471.485 & -21.60 & 0.69 \\
\hline 122703 & 472.509 & -19.21 & 2.01 \\
\hline 122703 & 473.455 & -15.39 & 0.69 \\
\hline 122703 & 474.464 & -17.16 & 0.94 \\
\hline 150453 & 471.521 & 0.82 & 0.69 \\
\hline 150453 & 471.620 & 0.47 & 0.69 \\
\hline 150453 & 472.586 & 0.12 & 0.28 \\
\hline 150453 & 473.541 & 0.64 & 0.48 \\
\hline 150453 & 474.568 & -0.10 & 0.28 \\
\hline 150453 & 506.486 & 1.54 & 0.28 \\
\hline 150557 & 471.510 & -49.75 & 0.48 \\
\hline 150557 & 472.492 & -49.42 & 0.69 \\
\hline 150557 & 473.496 & -48.36 & 0.90 \\
\hline 150557 & 474.494 & -49.64 & 0.69 \\
\hline 150557 & 474.540 & -50.22 & 0.48 \\
\hline 150557 & 505.532 & -48.20 & 0.48 \\
\hline 150557 & 506.412 & -48.49 & 0.94 \\
\hline 150557 & 506.467 & -50.15 & 0.28 \\
\hline 150557 & 509.393 & -49.60 & 0.48 \\
\hline 155646 & 470.552 & 59.76 & 0.48 \\
\hline 155646 & 471.535 & 61.08 & 0.48 \\
\hline 155646 & 472.542 & 62.36 & 0.48 \\
\hline 155646 & 473.518 & 59.78 & 0.28 \\
\hline 155646 & 474.524 & 58.64 & 0.48 \\
\hline 155646 & 505.545 & 60.05 & 0.48 \\
\hline 155646 & 506.445 & 59.95 & 0.28 \\
\hline 155646 & 506.547 & 60.70 & 0.28 \\
\hline 159561 & 470.565 & 10.69 & 0.69 \\
\hline 159561 & 471.543 & 11.10 & 0.48 \\
\hline 159561 & 472.550 & 12.23 & 0.69 \\
\hline 159561 & 472.631 & 10.25 & 0.94 \\
\hline 159561 & 473.527 & 12.03 & 0.90 \\
\hline 159561 & 474.510 & 10.82 & 0.69 \\
\hline 159561 & 505.431 & 11.46 & 0.90 \\
\hline 159561 & 506.457 & 10.65 & 0.48 \\
\hline 159561 & 507.608 & 9.68 & 0.69 \\
\hline 159561 & 509.446 & 8.29 & 0.48 \\
\hline 171856 & 470.639 & -5.56 & 2.01 \\
\hline 171856 & 473.620 & -5.96 & 1.34 \\
\hline 171856 & 506.584 & -21.70 & 0.48 \\
\hline 171856 & 508.544 & -19.14 & 0.69 \\
\hline 174866 & 470.625 & -39.39 & 0.94 \\
\hline 174866 & 472.615 & -38.50 & 0.69 \\
\hline 174866 & 473.606 & -40.45 & 0.48 \\
\hline 174866 & 505.585 & -40.89 & 0.90 \\
\hline 174866 & 506.571 & -43.56 & 1.34 \\
\hline 174866 & 509.521 & -42.08 & 0.69 \\
\hline
\end{tabular}

Table 3. continued

\begin{tabular}{|c|c|c|c|}
\hline HD & $\begin{array}{c}\text { HJD } \\
(+2449000)\end{array}$ & $\begin{array}{r}\begin{array}{r}V_{\mathrm{r}} \\
\mathrm{km} \mathrm{s}^{-1}\end{array} \\
\end{array}$ & $\begin{array}{c}I \\
\mathrm{~km} \mathrm{~s}^{-1}\end{array}$ \\
\hline$\overline{176971}$ & 470.575 & -34.62 & 0.90 \\
\hline 176971 & 471.553 & -34.28 & 0.48 \\
\hline 176971 & 472.559 & -31.63 & 0.69 \\
\hline 176971 & 473.560 & -35.13 & 0.48 \\
\hline 176971 & 474.579 & -33.63 & 0.69 \\
\hline 176971 & 474.625 & -34.88 & 1.34 \\
\hline 176971 & 505.461 & -34.60 & 0.94 \\
\hline 176971 & 506.517 & -32.51 & 0.69 \\
\hline 176971 & 509.414 & -29.47 & 1.78 \\
\hline 177392 & 470.608 & 10.13 & 0.48 \\
\hline 177392 & 471.577 & 9.51 & 1.56 \\
\hline 177392 & 472.603 & 12.38 & 0.69 \\
\hline 177392 & 473.580 & 10.12 & 0.90 \\
\hline 177392 & 474.593 & 6.75 & 0.90 \\
\hline 177392 & 505.481 & 11.21 & 0.90 \\
\hline 177392 & 506.502 & 10.31 & 2.01 \\
\hline 177392 & 508.525 & 6.75 & 0.90 \\
\hline 177392 & 509.459 & 8.69 & 0.28 \\
\hline 177392 & 509.539 & 7.37 & 0.90 \\
\hline 178187 & 470.600 & -24.85 & 0.69 \\
\hline 178187 & 471.563 & -24.43 & 0.69 \\
\hline 178187 & 472.568 & -24.82 & 0.48 \\
\hline 178187 & 473.570 & -25.11 & 0.94 \\
\hline 178187 & 474.602 & -24.69 & 0.90 \\
\hline 178187 & 505.440 & -24.69 & 0.69 \\
\hline 178187 & 505.518 & -25.04 & 0.90 \\
\hline 178187 & 506.531 & -25.03 & 0.90 \\
\hline 178187 & 507.585 & -22.70 & 0.48 \\
\hline 178187 & 508.506 & -23.06 & 0.69 \\
\hline 186005 & 472.623 & -40.39 & 1.34 \\
\hline 186005 & 474.634 & -39.98 & 0.90 \\
\hline 186005 & 506.593 & -39.50 & 0.90 \\
\hline 186005 & 509.593 & -42.63 & 0.48 \\
\hline 187764 & 471.598 & -8.02 & 0.69 \\
\hline 187764 & 473.589 & -5.96 & 0.48 \\
\hline 187764 & 474.551 & -8.39 & 0.90 \\
\hline 187764 & 474.612 & -4.71 & 0.90 \\
\hline 187764 & 505.560 & -6.14 & 0.69 \\
\hline 187764 & 506.554 & -3.10 & 0.69 \\
\hline 187764 & 509.477 & -4.64 & 0.69 \\
\hline 190172 & 471.628 & 2.82 & 0.48 \\
\hline 190172 & 473.628 & 6.00 & 0.28 \\
\hline 190172 & 506.602 & 3.11 & 0.48 \\
\hline 203842 & 663.228 & -25.94 & 0.69 \\
\hline 203842 & 665.246 & -27.74 & 0.48 \\
\hline 203842 & 690.313 & -26.43 & 0.69 \\
\hline 203842 & 692.296 & -24.73 & 0.90 \\
\hline 203842 & 693.243 & -23.22 & 0.48 \\
\hline 204577 & 663.245 & -10.45 & 0.48 \\
\hline 204577 & 693.273 & -8.69 & 0.69 \\
\hline 205852 & 663.262 & -31.90 & 0.90 \\
\hline 205852 & 692.307 & -31.83 & 0.69 \\
\hline 205852 & 693.308 & -32.48 & 0.69 \\
\hline 209166 & 663.275 & 5.22 & 0.28 \\
\hline 209166 & 692.314 & 6.36 & 0.28 \\
\hline 209166 & 693.322 & 6.82 & 0.48 \\
\hline
\end{tabular}


Table 3. continued

\begin{tabular}{rcrc}
\hline HD & $\begin{array}{c}\text { HJD } \\
(+2449000)\end{array}$ & $\begin{array}{r}V_{\mathrm{r}} \\
\mathrm{km} \mathrm{s}^{-1}\end{array}$ & $\begin{array}{c}I \\
\mathrm{~km} \mathrm{~s}^{-1}\end{array}$ \\
\hline 210516 & 663.295 & 10.46 & 0.69 \\
210516 & 692.332 & 11.38 & 0.94 \\
210516 & 693.339 & 12.16 & 0.94 \\
& & & \\
216701 & 663.314 & 12.70 & 0.48 \\
216701 & 692.352 & 15.26 & 0.69 \\
216701 & 693.355 & 11.36 & 0.48 \\
& & & \\
217131 & 663.334 & -12.12 & 0.28 \\
217131 & 693.374 & -11.35 & 0.48 \\
& & & \\
219891 & 663.352 & -3.04 & 0.90 \\
219891 & 689.338 & -3.01 & 2.01 \\
219891 & 693.396 & -0.48 & 0.94 \\
& & & \\
224995 & 663.383 & 8.13 & 0.48 \\
224995 & 689.302 & 7.14 & 0.90 \\
224995 & 693.431 & 7.48 & 0.69 \\
\hline
\end{tabular}

compute the residuals to the regression line fitting the cumulative distribution. The $\alpha$ parameter corresponding to the minimum residuals is then adopted. Figure 5 shows the behaviour of the rms deviation of the residuals as a function of $\alpha$. A minimum clearly appears around $\alpha=1.2$. This means that the error on $\sigma_{V_{r}}^{\mathrm{s}}$ is underestimated by about $20 \%$, which is quite reasonable considering the numerous uncertainties affecting its determination. Figure 6 shows the histogram and cumulative distribution of $P\left(\chi^{2}\right)$ for $\alpha=1.2$, as well as the straight line minimising the residuals of the cumulative distribution.

According to this criterion, $52 \%$ of the stars are variable and are listed in Table 4; the others are listed in Table 5. Each table gives the spectral type, $P\left(\chi^{2}\right)$, the blanketing parameter $\Delta m_{2}, v \sin i$ and eventually some remarks. The source of the projected rotational velocities is Abt \& Morrell (1995) or the Bright Star Catalogue (BSC), except for HD 6706, HD 122703, HD 150453, HD 190172 and HD 217131 whose $v \sin i$ is determined by the optimum fit of a synthetic spectrum to our observed spectra. The spectral types are taken from Hauck (1986) who refers to Cowley et al. (1969), Cowley (1976), the Michigan catalogue (Houk \& Cowley 1975; Houk 1978, 1982), Jaschek (1978) and the BSC. $\Delta m_{2}$ are taken from the Geneva photometry database. The values can differ from those of Hauck (1986) because new measurements have been made and a new reference sequence for the Hyades has been defined (see Sect. 5.1). The $\Delta m_{2}$ value can be weaker by a few thousandths of magnitude in the most unfavourable cases for visual doubles. This effect can only diminish the sample of metallic F giants, while the sample of metallic giants cannot be polluted by nonmetallic stars. Remarks D and SB come from the BSC.

The stars HD 2628 (3 measurements), HD 10845 (2), HD 11522 (2), HD 24832 (3), HD 62437 (4), HD 69997 (4), HD 1772392 (10) and HD 187764 (7) belong to the

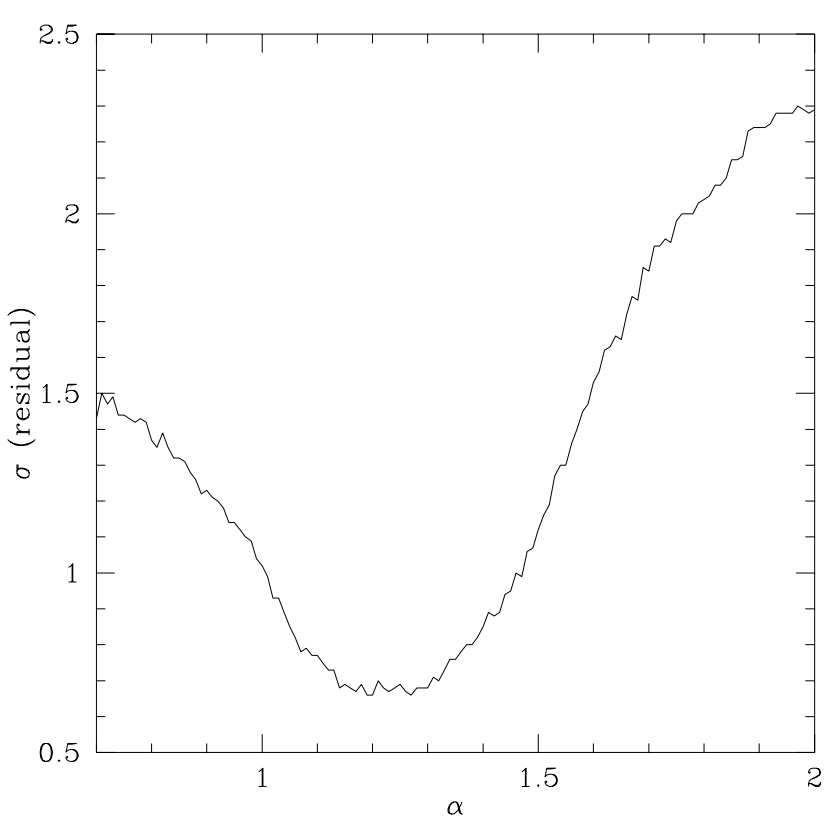

Fig. 5. Standard deviation of the residuals as a function of the $\alpha$ parameter

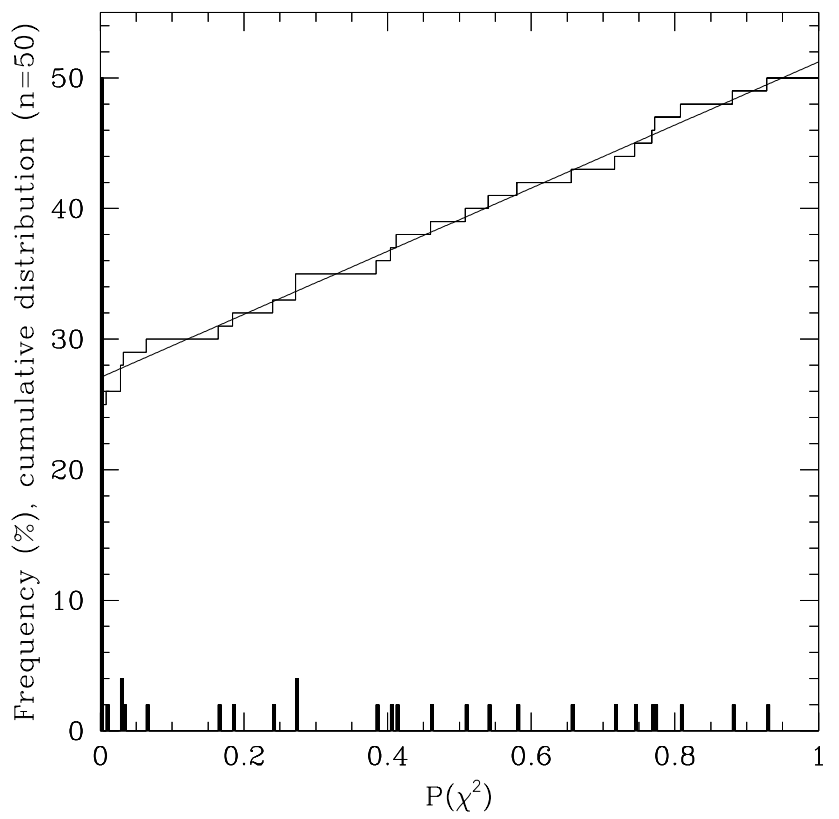

Fig. 6. Histogram and cumulative distribution of the $P\left(\chi^{2}\right)$ for the $50 \mathrm{~A}$ and $\mathrm{F}$ giant stars. These distributions correspond to an $\alpha$ parameter of 1.2 ensuring a flat distribution between 0 and 1 . We have also drawn the straight line determined by a least-squares fit of the cumulative distribution 
catalogue of $\delta$ Scuti stars of Rodriguez et al. (1994). In principle, all of these stars should be detected as variable, but the first three are not. For these, we have only a few measurements separated by several days. As ill luck would have it, for HD 2628 and HD 11522 the exposures are made at the same pulsational phase. For HD 10845, our measurements cover different phases, but the small amplitude of the lightcurve ( $0.02 \mathrm{mag}$ in the $V$ filter) is probably responsible for the non-detection. For the five $\delta$ Scuti stars detected, we find an average ratio of $110 \mathrm{~km} \mathrm{~s}^{-1} \mathrm{mag}^{-1}$ between the peak-to-peak radial velocity and photometric variations, which is compatible with the value of $92 \mathrm{~km} \mathrm{~s}^{-1} \mathrm{mag}^{-1}$ given by Breger (1979). Therefore, it seems that the $V_{\mathrm{r}}$ variation of these five objects is only due to pulsation and not to any orbital motion.

Among the stars not detected as variable, five are listed as SB in the BSC: HD 50019, HD 84607, HD 86611, HD 89025 and HD 92787. Low spectroscopic dispersion $\left(30-40 \AA \mathrm{mm}^{-1}\right)$ and fast rotational velocity may probably explain the large variations reported in the past. Figure 7 shows for these five stars the rms scatter of the radial velocities in the literature as a function of $v \sin i$. For HD 89025, we did not take into account the measurements made by Henroteau (1923), because they differ systematically from the others and would generate an artificially larger dispersion. For the older measurements, there is a clear correlation between dispersion and rotation: when $v \sin i$ increases from 70 to $215 \mathrm{~km} \mathrm{~s}^{-1}, \sigma$ increases from 7 to $25 \mathrm{~km} \mathrm{~s}^{-1}$. Our mean radial velocities values are compatible with the older ones, except for HD 86611 which rotates very fast.

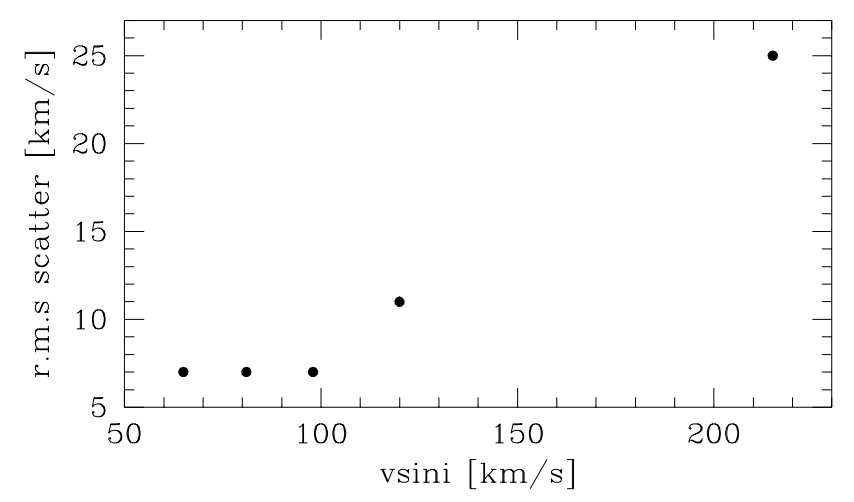

Fig. 7. Rms scatter of the $V_{\mathrm{r}}$ as a function of $v \sin i$ for stars considered as SB in the BSC but not detected variable in this paper

In Fig. 8, we show the behaviour of the external scatter $E$ (which is equivalent to the dispersion of the measurements) as a function of $v \sin i$ for the fifty giant stars and the seven standards of the programme. Black and open symbols represent respectively non variable and variable stars on the basis of the $P\left(\chi^{2}\right)$. A linear regression in-
Table 4. Detected variable stars

\begin{tabular}{rcrrrl}
\hline HD & Spectral type & $P\left(\chi^{2}\right)$ & \multicolumn{1}{c}{$\Delta m_{2}$} & $v \sin i$ & Remarks \\
\hline 4338 & F2III & 0.000 & 0.009 & 98 & D \\
24832 & F1V & 0.000 & 0.004 & 150 & $\delta$ Scuti \\
30020 & F4IIIp & 0.000 & 0.089 & 60 & SB \\
34045 & F2III & 0.001 & 0.021 & 67 & \\
60489 & A7III & 0.000 & 0.008 & 15 & \\
62437 & F0III & 0.000 & 0.008 & 35 & $\delta$ Scuti \\
69997 & F2III & 0.000 & 0.042 & 25 & $\delta$ Scuti \\
82043 & F0III & 0.000 & 0.003 & 51 & \\
100418 & F9III & 0.000 & -0.020 & 33 & \\
103313 & F0V & 0.000 & 0.009 & 61 & \\
104827 & F0IV-V & 0.000 & 0.002 & 38 & SB,D \\
118295 & A7-F0V & 0.000 & -0.003 & 135 & \\
122703 & F5III & 0.000 & 0.008 & 69 & \\
150453 & F4III-IV & 0.001 & -0.033 & 10 & \\
155646 & F6III & 0.000 & -0.034 & $\leq 10$ & \\
159561 & A5III & 0.000 & -0.019 & 210 & SB \\
171856 & A8IIIn & 0.000 & -0.001 & 110 & D \\
174866 & A7Vn & 0.001 & -0.012 & 150 & \\
176971 & A4V & 0.000 & -0.016 & 125 & \\
177392 & F2III & 0.000 & 0.030 & 120 & $\delta$ Scuti \\
186005 & F1III & 0.003 & 0.006 & 140 & SB \\
187764 & F0III & 0.000 & -0.003 & 85 & $\delta$ Scuti \\
190172 & F4III & 0.000 & -0.001 & 25 & \\
203842 & F5III & 0.000 & -0.006 & 84 & \\
209166 & F4III & 0.001 & 0.007 & $<20$ & D \\
216701 & A7III & 0.000 & -0.005 & 80 & \\
\hline
\end{tabular}

cluding only non-variable stars is also represented. This straight line is, as a first approximation, the mean internal error $I$ as a function of $v \sin i$ and agrees well with the values determined previously. Most of the variable stars clearly appear above this line and then we could also use it as criterion of variability.

$\delta$-Scuti type stars are represented by triangles, the most variable of them having an external scatter of about $1.8 \mathrm{~km} \mathrm{~s}^{-1}$, which is reasonable for stars with an amplitude of $0.05 \mathrm{mag}$. We can see that most of the variable stars have an external scatter below $1.8 \mathrm{~km} \mathrm{~s}^{-1}$ and so the origin of this variability remains ambiguous. Some of them are intrinsic variables not as yet classified $\delta$ Scuti. Only stars with $E \geq 2 \mathrm{~km} \mathrm{~s}^{-1}$ can be considered as binaries with a high probability.

\subsection{Rate of detection}

We have made a simulation to determine the rate of detected variable stars as a function of the period. For this, a sample of 1000 double stars with given periods was created as a first step. A flat distribution of the mass ratio was assumed (Mazeh et al. 1992) with primary components of A and $\mathrm{F}$ types $\left(1.5-3 M_{\odot}\right)$. The orbital elements $T_{0}, \omega$ and $i$ are randomly distributed, while the eccentricity is distributed according to Duquennoy \& Mayor (1991): when the period is less than 10 days, the orbit is assumed to be circular; for periods between 10 and 1000 days, the eccentricity is distributed following a gaussian with a mean equal to 0.3 and $\sigma=0.15$ (cases with negative eccentricity were dropped and replaced); for longer periods, the 
Table 5. Non-variable stars

\begin{tabular}{rcrrrl}
\hline \multicolumn{1}{c}{ HD } & Spectral type & $P\left(\chi^{2}\right)$ & \multicolumn{1}{c}{$\Delta m_{2}$} & $v \sin i$ & Remarks \\
\hline 1671 & F5III & 0.929 & 0.002 & 41 & \\
2628 & A7III & 0.510 & -0.004 & 18 & $\delta$ Scuti \\
6706 & F5III & 0.746 & -0.003 & 50 & \\
10845 & A9III & 0.770 & 0.015 & 85 & $\delta$ Scuti \\
11522 & F0III & 0.581 & -0.009 & 120 & $\delta$ Scuti \\
12573 & A5III & 0.275 & -0.017 & 95 & \\
17584 & F2III & 0.656 & 0.015 & 149 & \\
17918 & F5III & 0.461 & 0.033 & 120 & \\
21770 & F4III & 0.808 & -0.023 & 29 & \\
48737 & F5III & 0.065 & 0.008 & 70 & \\
50019 & A3III & 0.275 & -0.006 & 120 & SB \\
84607 & F4III & 0.388 & 0.023 & 98 & SB \\
86611 & F0V & 0.415 & -0.015 & 215 & SB \\
89025 & F0III & 0.242 & 0.038 & 81 & SB \\
92787 & F5III & 0.719 & -0.023 & 65 & SB \\
108382 & A4V & 0.881 & 0.006 & 65 & \\
150557 & F2III-IV & 0.031 & -0.009 & 67 & \\
178187 & A4III & 0.032 & -0.003 & 35 & \\
204577 & F3III & 0.036 & 0.008 & $\leq 15$ & \\
205852 & F3III & 0.775 & 0.020 & 155 & \\
210516 & A3III & 0.407 & -0.017 & 40 & \\
217131 & F3III & 0.166 & -0.016 & 66 & \\
219891 & A5Vn & 0.186 & -0.007 & 175 & \\
224995 & A6V & 0.543 & -0.009 & 90 & \\
\hline
\end{tabular}

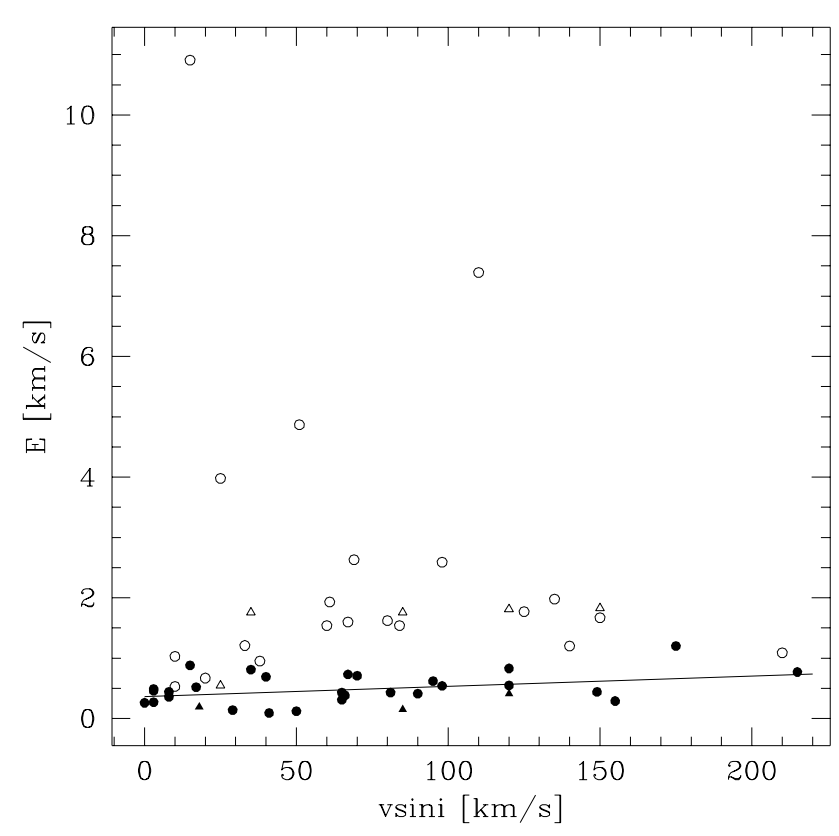

Fig. 8. External scatter $E$ as a function of $v \sin i$. Stars with $P\left(\chi^{2}\right)>0.01$ are represeted by black symbols and with $P\left(\chi^{2}\right) \leq 0.01$ by open symbols. $\delta$ Scuti type stars are represented by triangles. A linear regression is shown for constant stars distribution $f(e)=2 e$ is assumed. In a second step, the radial velocities of the created sample are computed at the epochs of observation of the real programme stars. Then a random internal error is added to these 50000 radial velocities. Finally the $P\left(\chi^{2}\right)$ value of each star is computed and we can take the census of detected binary stars for a given period. The results are presented in Fig. 9 for periods between 1 and $10^{5}$ days.

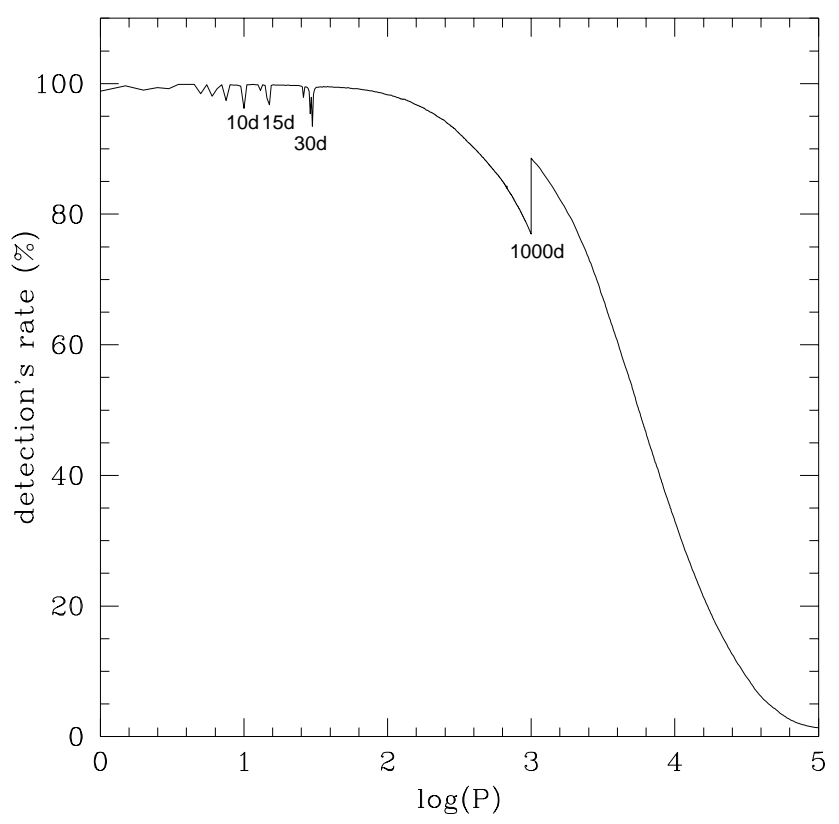

Fig. 9. Detection rate as a function of the period. $P$ is given in days

The simulated detection rate is very high for systems with periods below 100 days: it varies between $93.5 \%$ and 99.9\%. Above 100 days, the rate decreases rapidly, being about $80 \%$ for 1000 days and $30 \%$ for 10000 days. The discontinuity which appears for 1000 days is due to the strong change in the distribution of eccentricities: indeed, from this point on we grant more importance to large $e$. The size of this effect is related to the time distribution of the measurements. For instance, if the exposures were more distant in time, the discontinuity would be smaller. The simulation shows some very peaked depressions at shorter periods: at $P=30$ days, which corresponds to the time interval between two successive observing runs and at dividers of 30 , i.e. $15,10,7.5,6$ and 5 days. This is completely normal, because for such periods, the time distribution of the measurements makes the detection of binary stars less efficient. At $P=3$ days and $P=2$ days, the rate remains very high, because each run lasts for about 4 or 5 days. At $P=10$ days, there is a weak discontinuity due to the change of distribution of eccentricity, but this effect is hidden inside the peak. 
In addition, we have computed the mean rate of detection among binaries with periods less than 100 days. The binaries are created as before but the periods are distributed as a gaussian with a mean equal to $\overline{\log (P)}=4.8$ and $\sigma_{\log (P)}=2.3$ (Duquennoy \& Mayor 1991), where $P$ is given in days. When cut-offs at 1 and 100 days were imposed, the detection rate reached $99 \%$, i.e. all close binaries are detected. The rate remains as high as $94 \%$ for periods between 1 and 1000 days.

\section{Comparison between Am stars and F giants}

Before discussing the rotation and the rate of binaries among Am and giant A-F stars, it is useful to consider again the mean value of the blanketing parameter $\Delta m_{2}$ for Am stars as well as the range of spectral types of giant metallic stars.

\subsection{Parameter of metallicity $\Delta m_{2}$}

In the $B 2-V 1$ vs. $m_{2}$ diagram, the Am stars are located above the reference sequence defined by the Hyades (Hauck 1973). The difference $\Delta m_{2}$ is interpreted in terms of metallicity. Hauck \& Curchod (1980) have determined the mean value of $\Delta m_{2}$ for classical Am stars $(g \geq 5$, where $g$ is the difference between the spectral type deduced from the metallic lines and that from the $\mathrm{K}$ line) and for mild Am stars $(g<5)$ and have obtained respectively $0.013 \pm 0.019$ (146 stars) and $0.002 \pm 0.011(23$ stars). They considered only objects brighter than the 7 th magnitude in order to avoid any significant interstellar reddening.

Since then, a new sequence of reference has been defined (Hauck et al. 1991) taking into account new observations of the Hyades. This new sequence is different from the old one mainly for stars with a type later than $\mathrm{F}$; for early A type stars the old and new sequences are identical, while for late A and F stars they are separated by only a few thousandths of a magnitude. Taking into account the stars of the revised catalogue of Curchod \& Hauck (1979) brighter than the 7th magnitude, the new values are $\overline{\Delta m_{2}}=0.011 \pm 0.021$ (238 stars) and $\overline{\Delta m_{2}}=-0.001 \pm 0.025$ (101 stars) for classical and mild Am respectively, i.e a decrease of about 2 or 3 mmag with respect to the preceding values. Therefore, a giant star will be considered here as metallic whenever its $\Delta m_{2}$ is larger than or equal to 0.013 , rather than 0.015 as defined by Hauck \& Curchod (1980).

\subsection{Spectral types of metallic giants}

The diagram $\Delta m_{2}$ vs. $T_{\text {eff }}$ for giant A-F stars of Hauck (1986) is shown again in Fig. 10. The effective temperature is deduced from the semi-empirical calibrations of Künzli et al. (1997). Notice that only F stars have an enhanced $\Delta m_{2}$ value while this property never applies for A-type stars. Indeed, all stars with $\Delta m_{2} \geq 0.013$ have types between F0 and F6, except for HD 10845, HD 90277 and HD 147547 which are classified A9 and HD 4849 which is classified A9/F0. The lack of metallic stars later than F6 is explained by the diffusion theory: Vauclair \& Vauclair (1982) have defined the limit where the diffusion time scale for helium at the bottom of the surface convective zone equals the stellar lifetime in a $\log \left(L / L_{\odot}\right)$ vs $\log \left(T_{\text {eff }}\right)$ diagram; this limit crosses the area of giants at $\log \left(T_{\text {eff }}\right)=3.8$, which fits exactly the limit we find in our diagram. It is most interesting to notice that several metallic giants also are $\delta$ Scuti stars, because Am peculiarity and $\delta$ Scuti-type pulsation are mutually exclusive. Only mild Am stars may be $\delta$ Scuti, as well as $\delta$ Del stars. In this respect, the metallic $\mathrm{F}$ giants are completely similar to $\delta$ Del stars (Kurtz 1976).

The study of a relation between Am stars and metallic A-F giants is now restricted between Am and metallic giant F0-6 stars. In the next two section, we compare rotation and duplicity among these two samples.

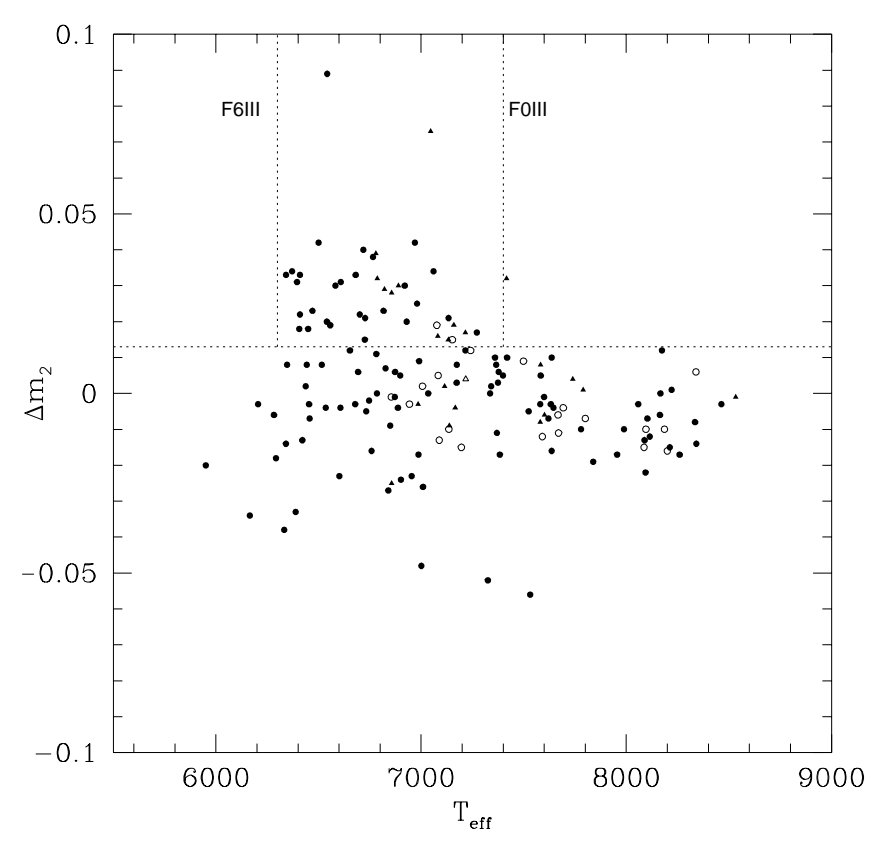

Fig. 10. $\Delta m_{2}$ vs. $T_{\text {eff }}$ diagram for A-F giants of Hauck (1986). The black dots represent spectroscopic giant stars and open dots photometric giants $(\Delta d \geq 0.12)$. $\delta$ Scuti stars are represented by triangles, the others by circles. The horizontal line separates metallic stars from normal ones. The two vertical lines at $6300 \mathrm{~K}$ and $7400 \mathrm{~K}$ define the red and blue limits for metallic giants

\section{3. $V \sin i$ of $A m$ and metallic F0-6III}

The Am stars are taken from the revised catalogue of Curchod \& Hauck (1979). Their $v \sin i$ used is an 
average of the values given by Abt \& Levy (1985), Uesugi \& Fukuda (1978, 1982), Bernacca \& Perinotto (1971), Boyarchuk \& Kopylov (1964). For stars with four measurements, we obtain a standard deviation of about $9 \mathrm{~km} \mathrm{~s}^{-1}$ which is a first indication of the precision of these $v \sin i$. The projected rotational velocities of metallic F0-6III stars are taken from Abt \& Morrell (1995) or the BSC (28 stars). Abt \& Morrell (1995) give an error on the determination of the radial velocity of about $10 \mathrm{~km} \mathrm{~s}^{-1}$. To make the comparison meaningful, we take into account only the Am stars brighter than the 7th magnitude and with $\Delta m_{2} \geq 0.013$ (98 stars). The Am stars with a low $\Delta m_{2}$ value have underabundant scandium and calcium, and normal or slightly overabundant heavier elements. On the other hand, F0-6III stars with low $\Delta m_{2}$ have a normal chemical composition, so there is no similarity between these two categories.

We present in Fig. 11 the histograms and cumulative distributions of the projected rotational velocities for the Am and metallic F0-6III stars, and the cumulative distribution for non metallic FIII stars. Obviously, Am stars (full line) and metallic F0-6 giants (dotted line) do not follow the same distribution. The $v \sin i$ of Am stars are below $100 \mathrm{~km} \mathrm{~s}^{-1}$ except for one star, while those of $\mathrm{F}$ giants are often faster than $100 \mathrm{~km} \mathrm{~s}^{-1}$. The maximum of the distribution for Am star is between 30 and $40 \mathrm{~km} \mathrm{~s}^{-1}$; for $\mathrm{F}$ giants, the distribution seems flat with a cut-off at about $160 \mathrm{~km} \mathrm{~s}^{-1}$.
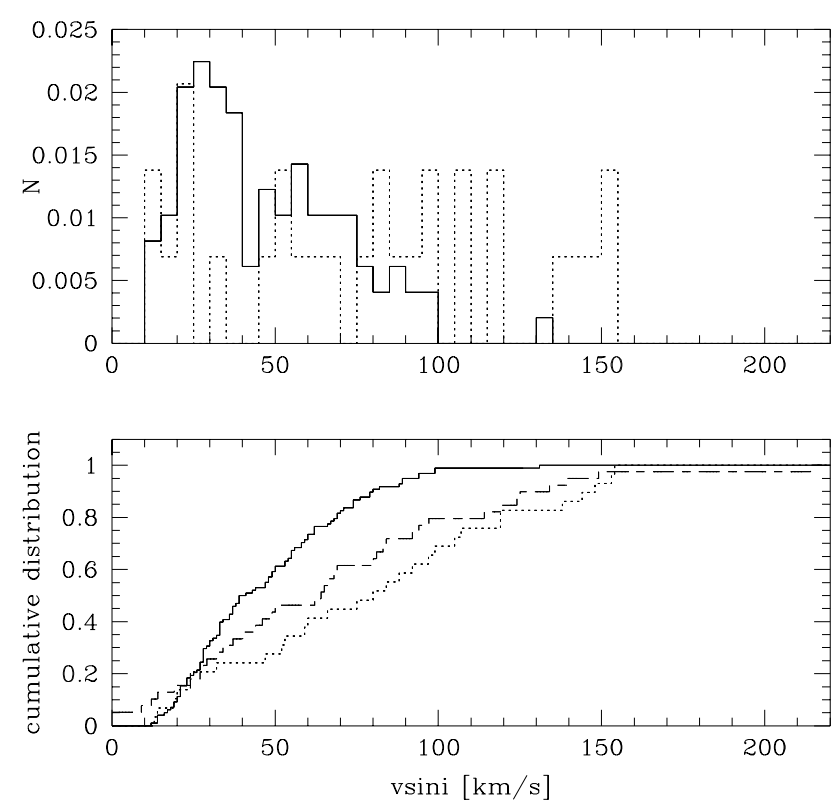

Fig. 11. Comparison between the distributions of $v \sin i$ for Am stars (full line) and metallic F0-6III stars (dotted line). We have also given the cumulative distribution for giant non-metallic F stars (broken line). Histograms and cumulative distributions are normalised
Cumulative distributions give more satisfactory information than histograms because they do not depend on the width of the bins. We have applied the KolmogorovSmirnov test to the distributions of the Am stars and of the metallic giants: adopting the $H_{0}$ hypothesis that they are identical, one obtains the probability $P=3.69210^{-4}$ to have a test value at least as extreme as the actually observed one. Therefore we can reject the $H_{0}$ hypothesis at the $99 \%$ confidence level and the distributions are very probably not similar. In the sample of $\mathrm{F}$ giants, some $\delta$ Scuti stars are present; in the framework of the scenario where Am stars are progenitors of metallic F giants, this implies that Am stars may pulsate as soon as they have evolved into giants. This assumption is based on the fact that some $\delta$ Del stars do pulsate and may be evolved Am stars (Kurtz 1976). If this assumption is wrong, then the sample of metallic giants is polluted. For that reason, we have applied the test used above to Am and $\mathrm{F}$ giants not known as $\delta$ Scuti stars and found $P=6.22710^{-4}$, which does not change the conclusion.

The Kolmogorov-Smirnov test applied to metallic and non-metallic FIII stars gives a $P$ value of 0.46 . So there is no real difference between these two samples from the point of view of projected rotational velocities, contrary to what we observe between Am and normal A dwarf.

\subsection{Rate of binaries among Am and metallic F0-6III}

Let us discuss now the rate of close binaries with periods below 1000 days among metallic F0-6 giants.

Since there are only 10 such stars in our sample of stars measured with Aurélie, we have to rely largely on results published in the literature. Table 6 lists some remarks about these data for each of the 41 metallic giants. For HD 4919 and HD 177482, however, the $V_{\mathrm{r}}$ data are too poor and inaccurate. Only 39 stars have therefore been retained, of which 6 stars are strictly speaking members of tight binaries: HD 30020, HD 34045, HD 43905, HD 85040, HD 108722 and HD 110318, which constitute $15.4 \%$ of the sample. For some other stars, the decision is less clear-cut. For fast rotators, it is difficult to know whether the observed dispersion is just due to the large $v \sin i$ or betrays an orbital motion; this is especially the case of HD 13174 and HD 147547. For the $\delta$ Scuti stars HD 214441, we observe a ratio of about $200 \mathrm{~km} \mathrm{~s}^{-1} \mathrm{mag}^{-1}$ between the peak-to-peak radial velocity and photometric variation which is double the value given by Breger (1979). Thus the additional variation in radial velocities could be due to orbital motion. But if these three stars are added to the sample of tight binaries, the proportion would only increase to $23.1 \%$ and to $23.3 \%$ if we take only $\mathrm{F}$ giants not known as $\delta$ Scuti type stars. These values are the observed rates; the real rates must be only slightly higher because the fraction of undetected stars is weak for small periods. It is interesting to note that the observed rate 
agrees with the value for solar-type stars in the vicinity of the Sun, i.e. 21.7\% (Duquennoy \& Mayor 1991) for $P \leq$ 1000 days.

Table 6. Discussion about the duplicity of all photometric metallic F giant stars of Hauck (1986).

HD 1324:

Single star (Evans et al. 1964).

HD 2724:

$\delta$ Scuti (Rodriguez et al. 1994). The $V_{\mathrm{r}}$ measurements of Nordström \& Andersen (1985) show no variation.

\section{HD 4849:}

$\delta$ Scuti (Rodriguez et al. 1994). The $V_{\mathrm{r}}$ measurements of Nordström \& Andersen (1985) show no variation.

\section{HD 4919:}

$\delta$ Scuti (Rodriguez et al. 1994). As we only have an old $V_{\mathrm{r}}$ from the catalogue of Campbell (1913), we cannot conclude about the duplicity.

HD 10845:

$\delta$ Scuti (Rodriguez et al. 1994). Our measurements show no variation.

HD 12311:

From his 15 exposures, Campbell (1928) indicated that this star is probably variable. At the time, he had only a few broad lines. Catchpole et al. (1982) observed no variation from 4 measurements. They obtained a mean radial velocity of $15.2 \pm 3.1 \mathrm{~km} \mathrm{~s}^{-1}$.

HD 13174:

Campbell (1928) obtained values covering a large interval $\left[-10,+20 \mathrm{~km} \mathrm{~s}^{-1}\right]$. This dispersion is probably due essentially to a fast rotation ( $v \sin i=154)$, nevertheless we cannot exclude a close companion. Adams et al. (1929) and Abt (1969) gave coherent velocities of about $-6 \mathrm{~km} \mathrm{~s}^{-1}$.

HD 15233:

The comparison between values obtained by Campbell $(1913,1928)$ and by Buscombe \& Morris (1958) shows that this star must be a binary with a long period. Indeed, these authors gave very different radial velocities: one found positive velocities, the others negative velocities, with a very good internal coherence if we consider the high rotation ( $\left.v \sin i=106 \mathrm{~km} \mathrm{~s}^{-1}\right)$. We find a value of $P\left(\chi^{2}\right)$ of 0.08 for the measurements of Buscombe \& Morris (1958) showing, according to this criterion, that no variation is detected on a scale of 700 days.

HD 17584:

Our measurements show no variation. We obtain a velocity of $18 \mathrm{~km} \mathrm{~s}^{-1}$ and Campbell (1913) $14 \mathrm{~km} \mathrm{~s}^{-1}$.

\section{HD 17918:}

Our measurements show no variation as is the case of the measurements of Shajn \& Albitzky (1932).

\section{HD 30020:}

The measurements made at OHP are compatible with the preceding ones of Adams et al. (1929) and Abt (1969). The available values are distributed between $34.40 \mathrm{~km} \mathrm{~s}^{-1}$ and $41.50 \mathrm{~km} \mathrm{~s}^{-1}$ with a characteristic time variation of about $0.7 \mathrm{~km} \mathrm{~s}^{-1} /$ day taking into account our measurements of December 1994. The variability is certainly due to an orbit because the photometric variations are less than $1 \mathrm{mmag}$ in the filter $B$ and $V$ of Johnson (Nelson \& Kreidl 1993).

\section{HD 33276:}

The measurements of Campbell (1928) and Abt (1965) show no variation.

\section{HD 34045:}

If we put together our measurements and those of Nordström \& Andersen (1985), the velocity varies between 26.32 and $34.70 \mathrm{~km} \mathrm{~s}^{-1}$. The characteristic time of variation is $0.95 \mathrm{~km} \mathrm{~s}^{-1} /$ day for our measurements (3 days between two measurments) and $0.91 \mathrm{~km} \mathrm{~s}^{-1} /$ day for Nordström \& Andersen (1985) (5 days between two measurements). As this star is not known as a $\delta$ Scuti, we think it is a tight binary.

\section{HD 40455:}

For this star, we have only 3 measurements of Nordström $\&$ Andersen (1985) on a scale of 300 days. The $P\left(\chi^{2}\right)$ is 0.74 and so this star is probably not a tight binary.

\section{HD 43905:}

SB1 with a period of 6.5 days (Mayor \& Mazeh 1987).

\section{HD 59881:}

The $P\left(\chi^{2}\right)$ is 0.06 and 0.13 for the measurements of Frost et al. (1929) et Penfold (1983) respectively. So this star is probably not a binary with a small period.

\section{HD 61064:}

If we put together the measurements of Campbell (1913, 1928) and those of Shajn \& Albitzky (1932), the mean value of the radial velocity is $45.72 \mathrm{~km} \mathrm{~s}^{-1}$ with a dispersion of $1.72 \mathrm{~km} \mathrm{~s}^{-1}$, so we observe no tangible variation.

\section{HD 61110:}

The measurements taken from Campbell (1913, 1928), Harper (1937), Buscombe \& Morris (1958), Abt (1969) give a mean radial velocity of $6.5 \mathrm{~km} \mathrm{~s}^{-1}$ and a dispersion of $3.5 \mathrm{~km} \mathrm{~s}^{-1}$ for 13 data. The dispersion is not so large if we consider the quite large rotational velocity $\left(90 \mathrm{~km} \mathrm{~s}^{-1}\right)$. So this star is probably not a tight binary.

\section{HD 69997:}

$\delta$ Scuti (Rodriguez et al. 1994). Our measurements allow to detect intrinsic variations but no orbital motion. 


\section{HD 79940:}

Single star (Evans et al. 1961). This star is noted SB in the BSC. This probably comes from Abt \& Biggs (1972). Indeed, in addition to the authors mentioned above who find a radial velocity of $2.2 \pm 1.5 \mathrm{~km} \mathrm{~s}^{-1}$, they list Campbell (1928, p. 143), who found a radial velocity of $12 \mathrm{~km} \mathrm{~s}^{-1}$ based on 3 measurements. The large rotational velocity probably explains the discrepancy.

\section{HD 84607:}

We detect no variation of this star at OHP. We obtain a mean radial velocity of $13 \mathrm{~km} \mathrm{~s}^{-1}$ which is compatible with the value of $16.3 \pm 2$ given by Shajn \& Albitzky (1932). Wilson \& Joy (1950) and Abt (1969) both found important fluctuations of velocity, which are spurious, probably due to the rotation. In both cases, the mean value is $13.4 \mathrm{~km} \mathrm{~s}^{-1}$. The SB notation in the BSC seems to be a premature decision.

\section{HD 85040:}

$\delta$ Scuti (Rodriguez et al. 1994). SB2 with a period of 4.14 days (Rosvick \& Scarfe 1991)

\section{HD 89025:}

The data since the beginning of the century show a radial velocity variation about $50 \mathrm{~km} \mathrm{~s}^{-1}$, but no short-term variation is detected, so it is probably not a tight binary as is suggested in the BSC. Indeed, apart from the values of Henroteau (1923) who obtained a mean velocity of about $-54 \mathrm{~km} \mathrm{~s}^{-1}$, the others have radial velocities between -30 and $0 \mathrm{~km} \mathrm{~s}^{-1}$ with a mean of $-17 \mathrm{~km} \mathrm{~s}^{-1}$. This scatter is probably due both to the quite large $v \sin i\left(81 \mathrm{~km} \mathrm{~s}^{-1}\right)$ and the low dispersion of the spectra $(\approx 30 \AA / \mathrm{mm})$. The computation of the $P\left(\chi^{2}\right)$ gives 0.88 and 0.53 for the measurements of Jones \& Haslam (1969) and Wooley et al. (1971) respectively, and 0 for the old measurements of Abt (1969). Our radial velocities are compatible with those of Adams et al. (1929) who obtained $-19.3 \pm 2.9$.

\section{HD 89254:}

The measurements given by Adams et al. (1929), Buscombes \& Morris (1958), Abt (1969) and Evans et al. (1961) suggest a constant radial velocity $\left(\overline{V_{\mathrm{r}}}=15.8 \pm 4.4\right)$. This dispersion is compatible with a rotational velocity of $76 \mathrm{~km} \mathrm{~s}^{-1}$.

\section{HD 90277:}

This star shows no variation. The mean velocity is $13.6 \mathrm{~km} \mathrm{~s}^{-1}$ with a dispersion of $2.7 \mathrm{~km} \mathrm{~s}^{-1}$ from the 36 measurements taken from 9 different authors.

\section{HD 90454:}

From the measurements of Cannon (1920) and those of Nordström \& Andersen (1985), the $P\left(\chi^{2}\right)$ is 0.31 and 0.03 respectively and so it is probably not a binary.

HD 105841:

Single star (Evans 1966).

\section{HD 108722:}

SB1 with a period of 17.954 days (Abt \& Levy 1976).

HD 110318:

SB2 with a period of 44.4 days (Sanford \& Karr 1942).

HD 115604:

$\delta$ Scuti (Rodriguez et al. 1994). On a time span of about 130 days, Smith (1982) observes a variation of radial velocity of $6.2 \mathrm{~km} \mathrm{~s}^{-1}$. Such a variation is probably due to pulsation only.

HD 125150:

Single star (Evans et al. 1964).

HD 126251:

Single star (Wilson \& Joy 1950).

\section{HD 147547:}

Radial velocities are distributed between -60 and $-30 \mathrm{~km} \mathrm{~s}^{-1}$ with a mean of about $-44 \mathrm{~km} \mathrm{~s}^{-1}$. The large dispersion is probably due to the fast rotation of $145 \mathrm{~km} \mathrm{~s}^{-1}$, nevertheless we cannot exclude a contribution of a companion.

\section{HD 157919:}

It shows no variation. The mean radial velocity is $37.2 \pm$ $2.4 \mathrm{~km} \mathrm{~s}^{-1}$ for 14 measurements taken from Campbell (1913), Campbell (1928), Shajn \& Albitzky (1932), Evans et al. (1957)

HD 177392:

$\delta$ Scuti (Rodriguez et al. 1994). Our measurements allow to detect intrinsic variations but no orbital motion.

\section{HD 177482:}

$\delta$ Scuti (Rodriguez et al. 1994). Campbell (1928) obtains values covering a large interval $\left[+3,+26 \mathrm{~km} \mathrm{~s}^{-1}\right]$ with the remark "poor lines" and Neubauer (1930) gives a value of $6.5 \mathrm{~km} \mathrm{~s}^{-1}$. With these old values, it is difficult to say anything about the duplicity.

\section{HD 181333:}

$\delta$ Scuti (Rodriguez et al. 1994). On a time span of about 38 days, Smith (1982) observes a variation of radial velocity of $4.7 \mathrm{~km} \mathrm{~s}^{-1}$. Such a variation is probably due to pulsation only.

\section{HD 196524:}

Binary star with a probable period of 26.65 years (Abt \& Levy 1976). So it will not be integrated in the sample of close binaries.

\section{HD 205852:}

From the $P\left(\chi^{2}\right)$ value, the measurements of Frost et al. (1929), Jones \& Haslam (1969) and this paper show no variations.

\section{HD 208741:}

Single star (Buscombe \& Morris 1958 and Evans et al. 1964). 


\section{HD 214441:}

$\delta$ Scuti with a variation of 0.05 mag in visual magnitude (Rodriguez et al. 1994). Nordström \& Andersen (1985) find a $\Delta V_{\mathrm{r}}$ of about $10 \mathrm{~km} \mathrm{~s}^{-1}$ and we then obtain a ratio of $200 \mathrm{~km} \mathrm{~s}^{-1} \mathrm{mag}^{-1}$ which is large in comparison with the value of $92 \mathrm{~km} \mathrm{~s}^{-1} \mathrm{mag}^{-1}$ given by Breger (1979). We cannot therefore exclude a companion for this star.

In their paper of 1985, Abt \& Levy estimated that the number of Am stars in double systems with periods less than 1000 days represents $75 \%$ of the sample, i.e. a rate considerably larger than what we find for metallic $\mathrm{F}$ giants. This is an additional reason to reject the possibility of any evolutionary link between Am stars and metallic F giants.

The upper limit of the fraction of binary stars among the forty non-metallic $\mathrm{A}$ and $\mathrm{F}$ giant stars measured at OHP is $47 \%$. This value is enhanced because it certainly includes some unrecognised intrinsic variables. Of the 113 stars of this category (Hauck 1986), 24 are classified as SB, 11 have a variable radial velocity $(V)$ and 6 are suspected variables $(V ?)$ in the BSC. The fraction of binaries with a small period among this type of stars is between $21.2 \%$ (SB only) and $36.3 \%(\mathrm{SB}+V+V$ ?) which may include some intrinsic variables. Thus frequencies of binaries with small periods among metallic and non-metallic $\mathrm{A}$ and $\mathrm{F}$ giant stars are not so different from each other, contrary to the case of Am and normal A dwarfs.

\section{Conclusion}

From the point of view of observed chemical abundances and of the theory of radiative diffusion, the scenario which considers Am stars as progenitors of metallic F0-6 giants is completely justified. It was worth the effort to try to settle this idea on firmer grounds, or alternatively to question it, by considering two other fundamental characteristics of Am stars, namely slow rotation and high rate of binaries. The main results of our work may be summarised as follows:

- Giant A-type stars never show an enhanced photometric metallicity, i.e $\Delta m_{2} \geq 0.013$. Therefore, if they are descendants of Am stars, they have not retained their chemical peculiarity. Most of them have probably never been Am in the past.

- One-third of the metallic F0-6 giants are fast rotators with $v \sin i>100 \mathrm{~km} \mathrm{~s}^{-1}$, while practically all Am stars are slow rotators with $v \sin i<100 \mathrm{~km} \mathrm{~s}^{-1}$. Moreover, the shape of the $v \sin i$ distribution is completely different in each case: for metallic F0-6III stars it is flat with a cut-off at about $160 \mathrm{~km} \mathrm{~s}^{-1}$, while that of Am stars shows a maximum between 30 and $40 \mathrm{~km} \mathrm{~s}^{-1}$ with a steady decrease towards $100 \mathrm{~km} \mathrm{~s}^{-1}$. This still holds valid when the giant $\mathrm{F}$ sample is restricted to stars not known as $\delta$ Scuti type.
- The non-metallic giants have the same $v \sin i$ distribution as the metallic ones.

- The rate of binaries among normal A-F giants is no more than $47 \%$ for orbital periods less than 1000 days. This fraction may be overestimated because part of the $V_{\mathrm{r}}$ variations observed might be due to $\delta$ Scutitype variations rather than orbital motion.

- For metallic F giants (considering or not $\delta$ Scuti stars), the best data in the literature together with our measurements indicate a rate of binaries with $P<1000$ days of less than $30 \%$. This is smaller, though not significantly so, than for normal giants. We may have missed binaries with a fast rotating primary, since the best $V_{\mathrm{r}}$ data in the literature concern sharp-lines stars (hence slow rotators). However, one sees no reason why most binaries should be in this case, especially as tidal friction would tend to slow down axial rotation. For Am stars, this rate is $75 \%$ (Abt \& Levy 1985).

It seems therefore difficult to admit that Am stars can be progenitors of metallic $\mathrm{F}$ giants. If such was the case, one should indeed expect:

1. A larger rate of binaries among metallic giants than among normal ones, its value being close to that of Am stars (75\%)

2. A $v \sin i$ distribution of metallic giants strongly peaked at very small values, with a tail extending to less than $100 \mathrm{~km} \mathrm{~s}^{-1}$. Indeed, the giants are all about to leave the main sequence and have, on average, larger radii than Am stars. Therefore they can only rotate more slowly, by conservation of angular momentum.

3. Widely different $v \sin i$ distributions for the normal, than for the metallic giants, as is the case for the normal A dwarfs compared with the Am stars.

None of these three expectations is fulfilled. As a whole, the metallic giants cannot be considered as evolved Am stars, although the previous considerations do not exclude the possibility that some of them (especially the slower rotators) may have been Am stars in the past.

One might object that even fast rotating metallic giants may have main sequence metallic progenitors, but the latter have gone unnoticed by the classifiers because of fast rotation. But such progenitors would have been detected by Geneva or Strömgren photometry (through the $m_{2}$ or $m_{1}$ parameters), while significant photometric metallicity is observed only in stars classified spectroscopically as Am, except for the metallic $\mathrm{F}$ giants. The assumption of overabundances remaining steady (apart from that of Ca) up to the very end of the main sequence life therefore seems wrong, and diffusion theory indeed predicts that they may change drastically on shorter timescales, at least near the ZAMS (Alecian 1996).

But if metallic $\mathrm{F}$ giants are not evolved Am stars, what is their origin then? The fact that they are not especially slow rotators is intriguing, because it suggests that no special initial conditions are required to produce them. The 
same can be said about the rate of binaries, which does not seem special either. In view of this, the very simplest alternative to the idea of Am progenitors is to speculate that every late A and early $\mathrm{F}$ star goes through a short phase of enhanced atmospheric metallicity around the end of its life on the main sequence.

We have seen that for some FIII stars the metallicity can coexist with high projected rotational velocity $\left(\geq 100 \mathrm{~km} \mathrm{~s}^{-1}\right)$. The explanation of this fact is a real challenge addressed to theoreticians of diffusion, because for main sequence stars metallicity can appear only in slow rotators $\left(\leq 100 \mathrm{~km} \mathrm{~s}^{-1}\right)$.

Acknowledgements. This research has made use of the Simbad database, operated at CDS, Strasbourg, France. We thank Drs. Michel Mayor and Stéphane Udry (Geneva Observatory) for providing us Coravel data for standard stars. We thank Mrs B. Wilhelm for the correction of the English text. This paper received the support of the Swiss National Science Foundation.

\section{Appendix}

\section{Importance of the choice of the limits for the fit of the $\mathbf{H} \beta$ line}

Simulations were carried out to observe the behaviour of $\overline{\lambda_{0}(\mathrm{H} \beta)}$ and $\sigma_{\lambda_{0}(\mathrm{H} \beta)}$ as a function of the choice of the two segments in order to find their optimal position and length.

Synthetic spectra were computed at $6000 \mathrm{~K}$ and $7500 \mathrm{~K}$ with a $\log g$ of 4 and solar metallicity. They were then convoluted by instrumental and rotational profiles. We considered projected rotational velocities of 50 and $100 \mathrm{kms}^{-1}$. Finally, a random noise was added, corresponding to a signal-to-noise ratio of 150 .

Two types of simulations were performed:

1. Two internal limits of the segments were fixed symmetrically with respect to the centre of the line, the external limits being moved away progressively from the line's centre. $\overline{\lambda_{0}(\mathrm{H} \beta)}$ and $\sigma_{\lambda_{0}(\mathrm{H} \beta)}$ was then computed for each position of these segments.

2. The external limits of the segments were fixed, the internal limits being brought regularly towards the centre of the line and $\overline{\lambda_{0}(\mathrm{H} \beta)}$ and $\sigma_{\lambda_{0}(\mathrm{H} \beta)}$ were again computed.

The results are given in Figs. 12 and 13, the upper part of them showing the variation of $\sigma_{\lambda_{0}(\mathrm{H} \beta)}$ and the lower part the variation of $\overline{\lambda_{0}(\mathrm{H} \beta)}$ as a function of the limits for the 4 spectra. Each curve corresponds to different initial conditions, i.e. to different fixed limits. The first type of simulation is represented by dotted lines and the second type by dots and dashes. The dispersion and the mean central wavelength are given as a function of the moving limit. To make these graphics easier to read, we have represented the right wing of the $\mathrm{H} \beta$ line with the scale in normalised flux (right axis of the graphics).
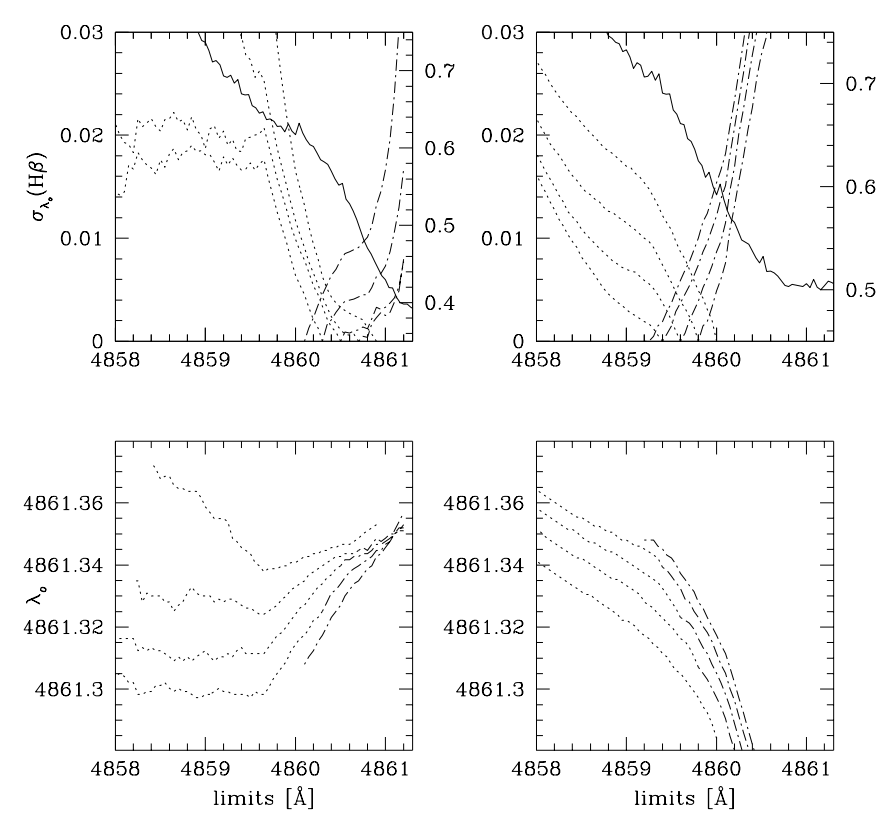

Fig. 12. Variation of $\overline{\lambda_{0}(\mathrm{H} \beta)}$ and $\sigma_{\lambda_{0}(\mathrm{H} \beta)}$ as a function of the limits for a star of $6000 \mathrm{~K}$ with a $\log g$ of 4 and a solar metallicity. The graph at the left simulates variations for a $v \sin i$ of $50 \mathrm{~km} \mathrm{~s}^{-1}$ and at the right of $100 \mathrm{~km} \mathrm{~s}^{-1}$. See text for comments
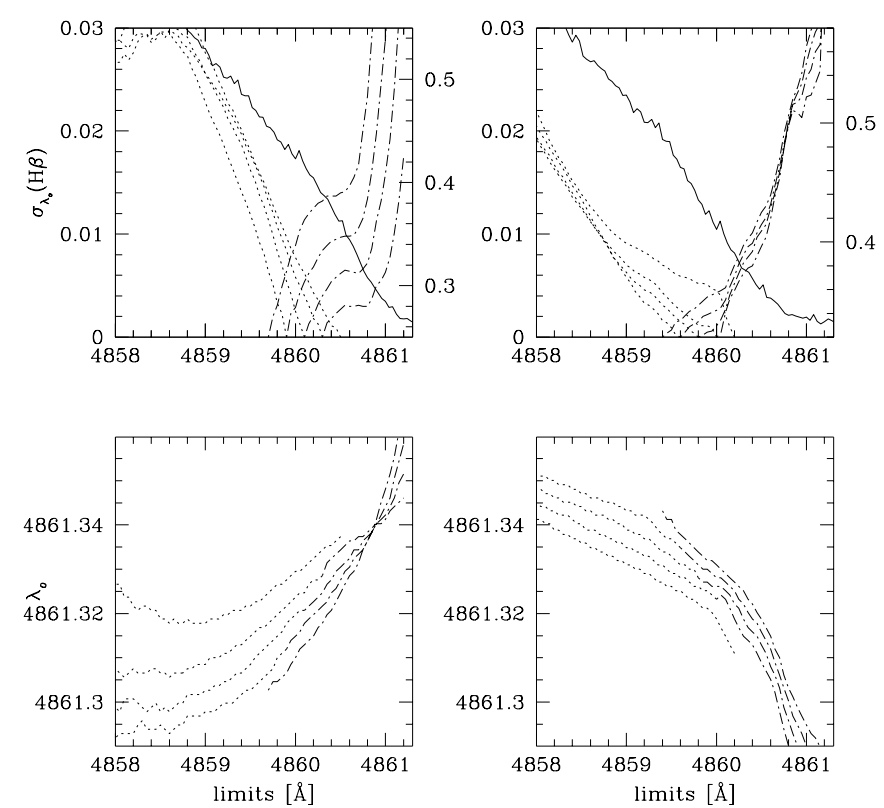

Fig. 13. Same as Fig. 11, but with $T_{\text {eff }}=7500 \mathrm{~K}$ 
First, let us discuss the behaviour of $\sigma_{\lambda_{0}(\mathrm{H} \beta)}$. When the internal and external limits are the same on each wing, the dispersion is evidently null, increasing when the internal and external limits are separated. This increase depends on each profile as seen in Figs. 12 and 13. Nevertheless, we can make general remarks. First, the nearer the limits to the centre of the line, the stronger the dispersion. This is easily understood, because near the centre only a few points are taken into account to make the fit and a weak change of limits leads to important changes of the three parameters of $L(\lambda)$. These parameters are not well defined either when we choose external limits too far from the centre, because $\mathrm{H} \beta$ have a lorentz profile only near the centre. Thus limits that are neither too close nor too far from the centre have to be chosen. Generally speaking, these subjective criteria are satisfactory when the segments lie on the linear part of the $\mathrm{H} \beta$ profile.

Increased $v \sin i$ and $T_{\text {eff }}$ make the determination of the central wavelength more difficult because the line is wider. The dispersion therefore increases with $v \sin i$ and $T_{\text {eff }}$. When the segments are on the linear part of the profile, the following dispersions result: at $6000 \mathrm{~K}, \sigma_{\lambda_{0}(\mathrm{H} \beta}$ varies from 0.003 to $0.007 \AA$ for $v \sin i$ equal to 50 and $100 \mathrm{~km} \mathrm{~s}^{-1}$ respectively and at $7500 \mathrm{~K}$, its values are 0.004 to $0.007 \AA$ for the same $v \sin i$. Thus the dispersion mainly depends on the rotational velocity and barely on the effective temperature.

On the lower graphics, one sees that $\overline{\lambda_{0}(\mathrm{H} \beta)}$ varies much as a function of the position and lengths of the segments. Nevertheless, if we consider only the linear portion, the variability of this parameter is not so important and corresponds to values given above. We observe that the shape of these fluctuations depends essentially on the $v \sin i$ : for a $v \sin i$ of $100 \mathrm{~km} \mathrm{~s}^{-1}, \overline{\lambda_{0}(\mathrm{H} \beta)}$ regularly decreases when the limits approach the centre of the line; for a $v \sin i$ of $50 \mathrm{~km} \mathrm{~s}^{-1}$ we observe an opposite trend.

The results of these simulations give only a qualitative idea about the behaviour of $\overline{\lambda_{0}(\mathrm{H} \beta)}$ and $\sigma_{\lambda_{0}(\mathrm{H} \beta)}$ as a function of the chosen segments. We have shown that these parameters are very sensitive to the limits, which have to be put on the linear parts of the line profile to alleviate this problem. For a given star, we always use the same limits in order to have a good internal coherence. Nevertheless, we may have systematic errors, but this is not a severe problem because we are interested in variations of radial velocity rather than in absolute values.

\section{References}

Abt H.A., 1965, ApJS 11, 429

Abt H.A., 1969, ApJS 19, 387

Abt H.A., Biggs E.S., 1972, Bibliography of Stellar Radial Velocities, Latham Process Corp., New York

Abt H.A., Levy S.G., 1976, ApJS 30, 273

Abt H.A., Levy S.G., 1985, ApJS 59, 229

Abt H.A., Morrell N.I., 1995, ApJS 99, 135

Abt H.A., Moyd K.I., 1973, ApJ 182, 809
Adams W.S., 1923, ApJ 57, 149

Adams W.S., Joy A.H., Sanford R.F., Strömberg G., 1929, ApJ 70, 207

Alecian G., 1996, A\&A 310, 878

Baranne A., Mayor M., Poncet J.-L., 1979, Vistas Astron. 23, 279

Bernacca P.L., Perinotto M., 1971, Contr. Oss. Astrofis. Univ. Padova 249

Berthet S., 1990, A\&A 227, 156

Berthet S., 1991, A\&A 251, 171

Berthet S., 1992, A\&A 253, 451

Boyarchuk A.A., Kopylov I.M., 1964, Publ. Obs. Crimee, 31, 44

Breger M., 1979, PASP 91, 5

Buscombe W., Morris P.M., 1958, MNRAS 118, 609

Campbell W.W., 1911, Lick Obs. Bull. 6, 140

Campbell W.W., 1912, Lick Obs. Bull. 7, 19

Campbell W.W., 1913, Lick Obs. Bull. 7, 113

Campbell W.W., 1928, Pub. of the Lick Obs. 16, 1

Cannon J.B., 1920, Pub. Dominion Obs. 4, 253

Catchpole R.M., Evans D.S., Jones D.H.P., King D.L., Wallis R.E., 1982, Royal Greenwich Obs. Bull. 188, 5

Charbonneau P., Michaud G., 1991, ApJ 370, 693

Cochran W.D., Hatzes A.P., Hancock T.J., 1991, ApJ 380, 35

Cowley A.P., 1976, PASP 88, 95

Cowley A.P., Cowley C.R., Jaschek M., Jaschek C., 1969, AJ 74,375

Curchod A., Hauck B., 1979, A\&AS 38, 449

Duquennoy A., Mayor M., 1991, A\&A 248, 485

Evans D.E., Menzies A., Stoy R.H., 1957, MNRAS 117, 534

Evans D.E., Menzies A., Stoy R.H.,Wayman P.A., 1961, Royal Greenwich Obs. Bull. 48, 389

Evans D.E., Laing J.D., Menzies A., Stoy R.H., 1964, Royal Greenwich Obs. Bull. 85, 207

Evans D.E., 1966, Royal Greenwich Obs. Bull. 110, 185

Frost E.B., Barrett S.B., Struve O., 1929, Pub. Yerkes Obs. 7, 1

Gillet D., Burnage R., Kohler D., et al., 1994, A\&AS 108, 181

Harper W.E., 1920, Pub. Dominion Obs. 4, 331

Harper W.E., 1934, Pub. Dominion Astrophys. Obs. 6, 151

Harper W.E., 1937, Pub. Dominion Astrophys. Obs. 7, 1

Hauck B., 1973, problems of calibration of Absolute Magnitudes and Temperature of Stars, IAU Symp. 54, 117

Hauck B., 1986, A\&A 155, 371

Hauck B., Curchod A., 1980, A\&A 92, 289

Hauck B., Jaschek C., Jaschek M, Andrillat Y., 1991, A\&A 252,260

Henroteau F., 1923, Pub. Dominion Obs. 8, 59

Hoffleit D., Jaschek C., 1982, The Bright Star Catalogue, 4th revised edition, Yale University Observatory, New Haven

Houk N., Cowley A.P., 1975, Michigan Catalogue of TwoDimensional Spectral Types for the HD Stars, Univ. of Michigan, Ann Arbor, Vol. 1

Houk N., 1978, Michigan Catalogue of Two-Dimensional Spectral Types for the HD Stars, Univ. of Michigan, Ann Arbor, Vol. 2

Houk N., 1982, Michigan Catalogue of Two-Dimensional Spectral Types for the HD Stars, Univ. of Michigan, Ann Arbor, Vol. 3

Jaschek M., 1978, A Catalogue of Selected MK Types, Inf. Bull. CDS 15, 121 
Jones H.P., Haslam C.M., 1969, Royal Greenwich Obs. Bull., 155

Jordan F.C., 1912, Pub. Allegheny Obs. 2, 121

Künzli M., North P., Kurucz R.L., Nicolet B., 1996, A\&AS 122,51

Kurtz D.W., 1976, ApJS 32, 651

Latham D.W., Mazeh T., Stefanik R.P., Mayor M., Burki G., 1989, Nat 339, 38

Mayor M., Maurice E., 1985, in: Stellar radial velocities, IAU Coll. No. 88, eds. A.G. Davis Philip and David W. Latham. Davis Press, p. 299

Mayor M., Mazeh T., 1987, A\&A 171, 157

Mayor M., Udry S., 1996 (private communication)

Mazeh T., Goldberg D., Duquennoy A., Mayor M., 1992, ApJ 401, 265

Michaud G., Charland Y., Vauclair S., Vauclair G., 1976, ApJ 210,447

Michaud G., Tarasick D., Charland Y., Pelletier C., 1983, ApJ 269, 239

Nelson J.N., Kreidl T.J., 1993, AJ 105, 1903

Neubauer F.J., 1930, Lick Obs. Bull. 15, 46

Nordström B., Andersen J., 1985, A\&AS 61, 53

North P., 1994, in: The 25th workshop and meeting of European working group on CP stars, Jankovics I. and
Vincze I.J. (eds.), Gothard Astrophysical Observatory of Eötvös University, Szombathely, Hungary, p. 3

0’Brien G.T., et al., 1986, ApJS 62, 899

Penfold J.E., 1971, PASP 83, 497

Plaskett W.E., Harper W.E., Young R.K., Plaskett H.H., 1921, Pub. Dominion Astrophys. Obs., 2, 1. Spencer Jones, H., 1928, CAPE 10-8, 95

Rodriguez E., Lopez de Coca P., Rolland A., Garrido R., Costa V., 1994, A\&AS 106, 21

Rosvick J.M., Scarfe C.D., 1991, PASP 103, 628

Sanford R.F., Karr E., 1942, ApJ 96, 214

Shajn G., Albitzky V., 1932, MNRAS 92, 771

Smith M.A., 1982, ApJ 254, 242

Tassoul J.-L., Tassoul M., 1992, ApJ 395, 259

Uesugi A., Fukuda I., 1978, Preliminary Catalogue of Rotational velocities, CDS

Uesugi A., Fukuda I., 1982, Revised Catalogue of Rotational Velocities, Uni. of Kyoto

Vauclair S., Vauclair G., 1982, ARA\&A 20, 37

Wilson R.E., Joy A.H., 1950, ApJ 111, 221

Wooley R., Penston M.J., Harding G.A., Martin W.L., Sinclair C.M., Aslan S., Savage A., Aly K., Assad A.S., 1971, Royal Greenwich Obs. Ann. 14, 1

Zahn J.P., 1977, A\&A 57, 383 